\title{
Contributions to a neurophysiology of meaning: The interpretation of written messages could be an automatic stimulus-reaction mechanism before becoming conscious processing of information.
}

Roberto Maffei, Livia S Convertini, Sabrina Quatraro, Stefania Ressa, Annalisa Velasco

Background. Even though the interpretation of natural language messages is generally conceived as the result of a conscious processing of the message content, the influence of unconscious factors is also well known. What is still insufficiently known is the way such factors work. We have tackled interpretation assuming it is a process, whose basic features are the same for the whole humankind, and employing a naturalistic approach (careful observation of phenomena in conditions the closest to "natural" ones, and precise description before and independently of data statistical analysis). Methodology. Our field research involved a random sample of 102 adults. We presented them with a complete real world-like case of written communication using unabridged message texts. We collected data (participants' written reports on their interpretations) in controlled conditions through a specially designed questionnaire (closed and opened answers); then, we treated it through qualitative and quantitative methods. Principal Findings. We gathered some evidence that, in written message interpretation, between reading and the attribution of conscious meaning, an intermediate step could exist (we named it "disassembling") which looks like an automatic reaction to the text words/expressions. Thus, the process of interpretation would be a discontinuous sequence of three steps having different natures: the initial "decoding" step (i.e. reading, which requires technical abilities), disassembling (the automatic reaction, an unconscious passage) and the final conscious attribution of meaning. If this is true, words and expressions would firstly function like physical stimuli, before being taken into account as symbols. Such hypothesis, once confirmed, could help explaining some links between the cultural (human communication) and the biological (stimulus-reaction mechanisms as the basis for meanings) dimension of humankind. 
1 Contributions to a neurophysiology of meaning: The interpretation of written messages could be an automatic stimulus-reaction mechanism before becoming conscious processing of information.

4 Roberto Maffei ${ }^{1^{*}}$, Livia Selene Convertini ${ }^{1}$, Sabrina Quatraro $^{1}$, Stefania Ressa ${ }^{1}$, Annalisa Velasco $^{1}$

$6{ }^{1}$ A.L.B.E.R.T. (ARPA-Firenze Landmarks on human Behaviour Experimental Research Team), Florence - Italy.

8 * E-mail: roberto@,robertomaffei.it ; albert@arpafirenze.it

\section{Introduction}

10 Human-environment interactions entail conscious knowledge, i.e. the attribution of

11 meanings (semantic aspect) to the incoming signals and stimuli. Interpretation, namely the

12 operation through which the meaning is attributed, is still widely unknown. A specific difficulty

13 is represented by natural language, although it has been studied almost since the dawn of

14 humankind, with researches ranging from the ancient rhetoric (for example, Geymonat, 1970;

15 Barthes, 1970; Perelman, 1977) to the most recent approaches complementing linguistics with

16 biology and neuroscience (for example Zuberbühler, 2005; Locke, 2009; $\underline{\text { Stekelenburg \& }}$

17 Vroomen, 2012). Messages are (or, at least, they appear) made up just of words; however, 
18 understanding a message always goes far beyond its words ${ }^{1}$. The available data does not give

19 definite answers to the researchers' questions; our field research intends to bring some

20 contributions to such endeavour.

21 Research lines and ideas: a synthetic overview. The available scientific literature is so

22 wide to prevent, inside the boundaries of our work, an exhaustive analysis. However, a rapid

23 survey is sufficient to reveal some trends, the first of which is the accelerating extension of these

24 studies towards the field of science. Another trend, thanks to the extraordinary development of

25 technology and informatics, is the enhancement of the studies that explore interpretation inside

26 the brain and its neural processes.

All this considered, we can roughly outline a picture with two main scientific research

lines, the first of which can be named Mind-centred approaches and can be synthesized as

follows. Understanding/interpretation is based on abstract (conceptual) knowledge. Incoming

information is provided through the body (perception) but the "mind"2 processes inputs at

symbolic level, turning them into propositional representations in the brain and understanding

them in terms of concepts. The answer to the inputs (reaction) is based on such comprehension

and is shaped as a command to some effectors (typically the motor system). Knowledge is the

result of a sort of computation; the mind is separated from the body and rules it. The role of the

motor system is totally passive.

\footnotetext{
${ }^{1}$ Material regarding the attempts to explain human communication and the questions of meaning and interpretation is really countless. Specific works will be indicated within the manuscript. Taking linguistics apart, we make reference to Pettigiani \& Sica, 2003 for a review (in Italian) of psychological main approaches; Krauss \& Fussell, 1996 for a wide survey from the perspective of social psychology.

${ }^{2}$ We will not enter the disputed question of mind, its existence, its nature and its relationships with the body in general and the brain in particular. For a first level of delving further into this subject: on the one hand, the early survey of Sperry, 1952; on the other hand, the more recent works of Marcus, 2004; Rose, 2005; Zeki, 2010. In the context of our Introduction, the "mind" is simply intended as a factor which, by following some theoretical positions, totally controls body through functions that differ from biological processes.
} 
38 body that instantiates understanding or, at least, co-exists with conceptual knowledge. When an

39 external stimulus/signal is perceived, it is firstly "understood" through a motor reaction which is

40 automatic, involuntary and based on "mental maps" that are motorial, not (or not only)

41 propositional. Understanding is a sort of motor experience that goes along with conscious

42 (rational) information processing; the body is not detachable from the mind and can drive it. The

43 role of the motor system is active and decisive for understanding.

44 The main features of the first group theories are synthesized in some recent works, for

45 example, Zipoli Caiani, 2013 (Chapters 1 and 2); Ferrari \& Rizzolatti, 2014 (specially Pag. 2);

46 Gallese, 2014 (specially Pag. 2, with the concept of ontological reductionism); Pulvermüller et

47 al., 2014 (specially Introduction and Fig. 1) $)^{3}$. The range of these theories goes from the merely

48 mechanical (and naïve) hypotheses of psychoneural isomorphism (Sperry, 1952, pp. 293-294),

49 and those inspired by the first electronic computers (Newell, Shaw \& Simon, 1958), to the

50 various I.P. (information processing) models (Massaro \& Cowan, 1993) and current cognitive

51 science positions (Negri et al., 2007; Mahon \& Caramazza, 2008; Mahon \& Caramazza, 2009).

52 The shared concept is that information is essentially processed in a linear and unidirectional

53 sequence, based upon a functional (besides the anatomical) separation among sensory,

54 associative and motor areas of the brain cortex (for a general presentation and discussion see also

55 Rizzolatti \& Sinigaglia, 2006, Chapter 1, specially pages 20-22; for a synthesis of the cognitivist

56 paradigm see Gallese, 2000, page 27).

The second group of theories (the body-centred ones) can be traced back, at least, to

58 XIX $^{\text {th }}$ Century, up to the works of Lotze, 1852 (cited in $\underline{\text { Rizzolatti \& Sinigaglia, 2006) and James, }}$

591890 , which present reflections on the relationships between perception and action. Other

${ }^{3}$ The last three works (Ferrari \& Rizzolatti, 2014; Gallese, 2014; Pulvermüller et al., 2014) are ascribable to the theories of the second group; nonetheless, they are cited also here because contain particularly clear syntheses of the opposite field positions. Ahead in the text we will describe a mirror-case (Hickok, 2009). 
60 philosophers came after ${ }^{4}$, up until a new series of neurophysiological studies appeared in the

61 second part of $\mathrm{XX}^{\text {th }}$ Century ${ }^{5}$. Such researches gathered evidence that the sequential processing

62 theory and the supposed motor system passive role are untenable. A leap ahead has probably

63 been accomplished with the discovery of mirror neurons (i Pellegrino et al., 1992) and the

64 related following studies (for example Gallese, 2000; Rizzolatti \& Craighero, 2004; $\underline{\text { Iacoboni et }}$

65 al., 2005; Rizzolatti \& Sinigaglia, 2006). According to this theory, understanding would be firstly

66 attained through a motor reaction of the body, "immediately and automatically" . Cognition

67 would be "embodied".

Embodiment of cognition, and its consequences on knowledge and interpretation process,

are the object of a lively scientific debate well exemplified in Hickok, 2009 (direct reference to

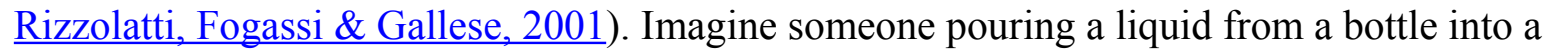

glass: by following the embodied cognition hypothesis, an observer can "embodily" understand

such action since, thanks to his mirror neurons, he undergoes a motor reaction "as if" himself

was actually pouring (by the way, such reaction does not turn into any actual movement, it

remains virtual). However, that pouring "could be understood as pouring, filling, emptying,

tipping, rotating, inverting, spilling (if the liquid missed its mark) or defying/ignoring/rebelling

(if the pourer was instructed not to pour)..." (see Hickok, 2009, page 1240, italic by the author).

Such example, in our opinion, well represents the crucial point: the scientifically evident

automatic reaction that instantiates embodied cognition does not explain the whole process of

${ }^{4}$ Some special mentions about the philosophers: Mach, 1897, in particular pages 1-8 (on the relationship between scientific knowledge and perceptual experience of physic world), pages 15-17 (a famous example on subjectivity of perspective) and pages 93-95 (sense organs as active elements of perception, fine-tuned through experience, rather than as passive receptors); Poincaré, 1902 [2003], especially Chapter 4 (on the relations between geometrical space and "representative", i.e. perceptual, space); Poincaré, 1908 [1997], Part I, specially pages 52-63 (phenomenology of a mathematical discovery and the role of sensitivity and aesthetic feeling); Merleau-Ponty, 1965, particularly Part II (with special regards to introduction chapter, on the impossibility to have a knowledge of the environment that is independent of the body experience).

${ }^{5}$ Some special mentions: Sperry, 1952, especially pages 299-300 on the relationships among perceptions and ideas; Jeannerod et al., 1995; Liberman \& Wahlen, 2000; Fowler, Galantucci \& Saltzman, 2003.

${ }^{6}$ We are intentionally employing the words "immediately and automatically": they are typically used in describing the mirror-system working. 
79 interpretation, and the attribution of a conceptual meaning seems to have a different nature. Thus,

80 we have either scientific evidence of embodied cognition or daily-life experience of scattered

81 conceptual interpretations; can these two visions be conciliated or are they alternative? And

82 which one can actually account for the field observations?

83 In the few last years, the hypotheses based on the mirror neurons discovery have been

84 refined, for example through the concepts of Mirroring mechanisms (MM) and Embodied

85 simulation (ES) (Gallese, 2005, 2006, 2007, 2008, 2009a; Gallese et al., 2009; Gallese \&

86 Sinigaglia, 2011a; Ferri, Gallese \& Costantini, 2011; Marino et al., 2011; Gallese \& Sinigaglia,

87 2012; Ferrari \& Rizzolatti, 2014; Gallese, 2014). About the ongoing dispute, a summary and a

88 state-of-the-art outline can be found in Zipoli Caiani, 2013 and one of the most interesting

89 documents is a forum (Gallese et al., 2011) inside which the most delicate and controversial

90 questions are widely debated.

91 Experimental research involving language. Theoretically, the divergence between

92 cognitivist and embodied cognition approaches can be synthesized as follows (for further

93 reference see, for example, Bedny et al., 2008; Rizzolatti \& Fabbri-Destro, 2008; Goldman \& de

94 Vignemont, 2009; Gallese, 2011; Gallese \& Sinigaglia, 2011b; Bedny et al., 2012): cognitivism

95 upholds the sequential processing idea, i.e. cognition would be the result of perception (the sound

96 of a spoken message as well as the sight of written words) followed by the symbolic processing

97 of what perceived (turning the spoken or written words into their meanings) followed by a

98 reaction (typically, but not exclusively, a motor one). Oppositely, the embodiment theories

99 uphold the concept of direct connections among cortical sensorial and motor areas

100 ("sensorimotor grounding" of cognition, Guan et al., 2013). Namely, the perceived spoken or 
101 written words would trigger a motor reaction and would be mentally represented also in a motor,

102 rather than a purely conceptual, way. In this sense, cognition would be embodied ${ }^{7}$.

103 From a technical slant, the two research lines tend to privilege different laboratory

104 approaches: cognitivist field frequently engages the noun-verbs dissociation problem, studying it

105 through researches on cortically damaged, selectively impaired patients (for example Crepaldi et

106 al., 2006; Arévalo et al., 2007; Moseley \& Pulvermüller, 2014; Gallese, 2014). Conversely, the

107 embodied cognition theorists mainly go searching for the connections between language and its

108 motor correlates, one well-known of which is the ACE (Action-sentence Compatibility Effect,

109 for example Vitevitch et al. 2013; Horchak et al., 2014). Such studies are frequently carried out

110 through neuroimaging works (for example Tettamanti et al., 2005; Aziz-Zadeh et al., 2006; Speer

111 et al., 2008; Aziz-Zadeh \& Damasio, 2008).

112 It is interesting to note that, beyond their important differences, both cognitivism and

113 embodiment research use, in laboratory experiments, words and short phrases isolated from

114 every contexts (see, for example, Bedny et al., 2008; Bedny et al., 2012, especially the Method

115 sections; for some critical reflections about the question, Pulvermüller et al, 2014, specifically

116 Pag. 80, Chapter 7). Such approach entails that meaning is an intrinsic feature of words,

117 something embedded inside them, and that interpretation consists in extracting it (actually, the

118 verb "to extract" is overtly used in scientific publications, for instance Mahon \& Caramazza,

119 2011).

120 About some recent trends. In the end, it is worth mentioning a recent specialised research

121 field inside psychophysics, in which researchers investigate cognition and semiosis through

122 probabilistic models (Chater, Tenenbaum \& Yuille, 2006; Ingram et al., 2008; Tenenbaum et al.,

${ }^{7}$ Such embodiment, inside the same embodied cognition field, can be conceived in different ways: it can stand alone, per se resolving the problem of knowledge ("sensorimotor processing underlies and constitutes cognition", Guan et al., 2013), or can be a "motor representation" that accompanies conscious knowledge processes (the two kinds of knowledge proposed by Gallese, for example in Gallese et al., 2011; see also Gallese, 2014). 
123 2011), applying the Bayesian inference to reproduce mental processes and to describe them

124 through algorithms (슬

125 Eldar, 2009; Arecchi, 2010c; Perfors et al., 2011; Fox \& Stafford, 2012). Such concepts are

126 currently in use also in the Artificial Intelligence (AI) field ${ }^{8}$; in addition, some studies make

127 reference to deterministic chaos (Guastello, 2002; Arecchi, 2011) and some others to Gödel's

128 incompleteness theorem as a limit to the possibility of understanding cognition "from inside"

129 (given that, while studying cognition, we become a system that investigates itself) 9

130 Methodological aspects and our approach. There are two main reasons why the question

131 of interpretation and meaning has not yet been scientifically solved, the first of which is that

132 there are still structural obstacles of technical and ethical nature ${ }^{10}$. The second main reason is the

133 complexity of natural language (its "equivocal" nature, see De Mauro, $2003^{11}$ ), which is usually

134 overcome through studying interpretation isolated from the interpreting organism and employing

135 simple stimuli (for instance Bedny \& Caramazza, 2011).

${ }^{8}$ The origins of Artificial Intelligence (AI) studies can be traced back to the Thirties and the works of Alan Turing on a possible "intelligent machine". About the origins, see Leavitt, 2007, chapters 6 and 7, and Turing, 1950 (the original work of Alan Turing). About the "Turing test" (testing the ability of distinguishing humans from computers through exchanging written messages) see a journalist's account in Christian, 2012. Some materials about recent research lines, closer to our article's topics (like machine learning and natural language or image interpretation), can be found in Mitchell, 1997; Menchetti et al., 2005; Mitchell, 2009; Khosravi \& Bina, 2010; Verbeke et al., 2012.

9 See Goldstein, 2006 for a popular-scientific coverage about Gödel and his theorem; Leavitt, 2007, chapters 2 and 3 , for a particularly clear synthesis of the theorem and its genesis (in connection with the Entscheidungsproblem, i.e. the "decision problem").

${ }^{10}$ About the technical difficulties of data collecting: experimental techniques used on macaque monkeys (electrode direct insertions inside single neurons) return very accurate measurements, but on small brain cortex surfaces. About the ethic difficulties: those techniques are almost impossible to be used on humans, and only indirect techniques as fMRI (functional Magnetic Resonance Imaging), MEG

(Magnetoencephalography), PET (Positron Emission Tomography) or TMS (Transcranial Magnetic Stimulation) are systematically employed. They cover wider brain cortex surfaces but with inferior accuracy; moreover, they present difficulties with regards to instrument positioning and image interpreting. For a survey of these difficulties see Rizzolatti \& Sinigaglia, 2006, chapters 2, 6, 7, and Rizzolatti \& Vozza, 2008, passim. A recent line of research is investigating the connections among single neurons activity and the total effects detectable through indirect techniques (see Iacoboni, 2008, chapter 7). In addition to all this, data interpretation and comparing are intrinsically difficult, given the differences in macaque and human brain cortex and the associated problem of identifying reliable correspondences.

${ }^{11}$ De Mauro, 2003 states that natural language is "equivocal" in etymological sense: from Latin aeque vocare (to name [different things] in the same way). That means: a same word can be used to refer to different meanings and different words can be used to indicate the same meaning. 
136 In field experiments, researchers who capitalise on the existence of mirror neurons

137 intentionally favour a naturalistic-like approach, letting the observed macaque monkeys freely

138 interact with available objects, rather than stimulate them with selected artificial stimuli only

139 (Rizzolatti \& Sinigaglia, 2006, p. 3; in addition, about the reductionism question and the

140 distinction between methodological and ontological reductionism, see Gallese, 2000, p. 26, and

141 Gallese, 2009b; Gallese, 2010). However, their approach has been also criticized (Pascolo \&

142 Budai, 2013). About the naturalistic-like approach, we had in our background two works about

143 interactions inside online collaborative groups (Maffei, 2006; Maffei, Cavari \& Ranieri, 2007)

144 which let us appreciate the potential of scientific observation in real world-like conditions.

145 On these bases we set up our approach. We set two objectives for our research: (i) To

146 understand the process of interpretation (i.e. how messages in natural language are turned into

147 meanings by receivers) as it works in real conditions, and design a structural model in order to

148 adequately represent it; (ii) To produce a first check of the formulated hypothesis. We tried a

149 naturalistic approach; this means, first, that a phenomenon must be carefully observed and

150 precisely described in conditions the closest to "natural" ones (natural conditions = the way and

151 the contexts in which the phenomenon usually manifests). Second, it means that observation and

152 description must precede analysis, being carried out independently of it. In such approach, the

153 role of the observers is critical, either if they are involved in or external to the phenomenon. In

154 our research, we have employed 102 observers of the first kind (the sample) and 5 (the authors)

155 of the second one; this way, we have collected 102 self-reports (participants' answers to a

156 specially designed questionnaire) and worked out one analytical report (our research) about 157 interpretation.

\section{Method}


Our work is not a clinical trial and no experimentations on the participants took place. In

160 addition, no personal data was collected or anyhow involved in the survey, and verbal informed

161 consent was requested to participants and obtained on the basis of a written presentation of the

162 survey and its modalities. The Ethics Committee for Scientific Research of the Association

163 ARPA-Firenze gave its approval either to the research design or to the informed consent

164 procedure. Further details related to method, sampling and ethical aspects can be found in the

165 Supporting Information (SI, from now on), Sections 0, 1 and 3. assumptions: first, interpretation is a process, rather than a single operation; second, the process

168 has the same basic (structural) universal characteristics. The rationale of our sampling was based

169 on such assumptions: according to our objectives, we focused on the reconstruction and

170 understanding of the process, rather than on sample features. Thus, the sample representativeness

171 (for example with respect to Italian people), as well as its social feature balance, were less

172 critical; from an extreme point of view, it could be sufficient that the sample members would 173 belong to human species. Operatively, we gathered our random sample through selecting only

174 Italian language native speakers, all adult; we strived to reach a reasonable balance about gender 175 and student/worker conditions. Further details (the procedure we used to randomize the sample

176 included) can be found in SI, Section 6; the results are presented in Tables 1-3.

177 The total sample (Table 1) results slightly imbalanced with regards to gender (women

178 exceed men), education (Graduates/Post-graduates exceed High-school degree granted

179 members) and employment (students/unemployed exceed employed members). For these

180 reasons, even though social features balance is less relevant in our work, we have selected more

181 homogeneous sub-samples from the total sample, in order to verify our analyses every time it

182 turned out necessary. The first sub-sample (“AGE”, Table 2) is exclusively composed by people 
183 over 29 years-old (60 members); the second one ("EMPLOYMENT", Table 3) is exclusively

184 composed by employed people (65 members).

185 Materials and procedure /2: the case. The main operative instruments through which we

186 have implemented our naturalistic-like approach (further details in SI, Section 0) are the case and

187 the questionnaire. We challenged our randomly selected sample of 102 adults with a real world-

188 like written communication case, using complete and unabridged message texts and collecting

189 participants' interpretations. The case we submitted to the sample (it is fully detailed and

190 documented in SI, Sections 2, 4 and 5) is a fictional piece very close to some real cases the

191 authors had professionally dealt with (the messages are drawn from actual messages and the

192 outlined relationship between the characters has been actually observed). Exactly, this case is an

193 online (via e-mail) interaction between two colleagues (no previous relations between them)

194 having different roles and ranks in the same organization; the two characters are a female

195 employee (XX) and a male professional (the "architect" YY, Project Account for the installation

196 of a heating plant in XX's office). Their interaction consists (from its start to its end) in

197 exchanging 5 e-mails, 3 of which (Messages \#1,\#3 and \#5) are sent by XX, which starts and

198 ends the interaction, and 2 (Messages \#2 and \#4) by YY. Such exchange (whose subject is the

199 work-in-progress of the heating plant) can be divided into two phases, during the first of which

200 (Messages \#1,\#2 and \#3) a conflict emerges that will be solved through a special version of the

201 fourth message (sent by YY); the solution of the conflict is confirmed by the last (fifth) message,

202 in which XX declares her satisfaction. A synthesis of the first three messages is the following

203 (further details and a full documentation can be found in SI, Section 4):

$204 \quad$ Msg \#1 (XX to YY) - A 67 word e-mail to the Project Account about the installation of the

205 heating plant in her office. She requires an inspection, claiming about "flaws" in the

206 present state of works. Flaws are no better detailed. She also declares she is speaking on 
behalf of some colleagues and uses the expression: "we would be pleased if, at least once, someone of our Corporation could come here and control...".

Msg \#2 (YY to XX) - A brief (48 words) answer of the Project Account in which the regularity of the Project progress is declared. The message ends with the phrase: "at the moment, the progress substantially complies with the chronogram”. Msg \#3 (XX to YY) - A 136 words reply in which XX declares herself totally unsatisfied. Her message presents two main features: (i) some minor flaws are listed; (ii) she expresses what resembles an actual threat against YY, in the case he would not take measures (she specifically refers to a hypothetical "waste of public money", given that the Project funding involved public resources).

218 (namely, he prepares the first version, the " $\mathrm{H}$ " one, of Msg \#4). The label " $\mathrm{H}$ " has been used 219 because such version is a "hard" reply; a YY's colleague suggests him a softer version (the "S" one) in order to avoid exacerbating the conflict. YY accepts the advice, he sends the Softer Msg

$221 \# 4(\mathrm{~S})$ to $\mathrm{XX}$ and the case ends with the conflict resolution (XX's satisfaction declared in Msg \#5). Full-text versions of the Hard Message \#4(H), the Softer \#4(S) and of Msg \#5 are displayed in Table 4; see also SI, Section 5 and Tables S1, S2, for details about the rationale of the two 224 alternative messages. interaction structure; in the first phase (Questions \#1 and \#2), we asked participants to interpret 
232 message submitting included) the two versions of Msg \#4 and asked them (Questions \#3 and \#4)

233 to give their separate interpretations. Finally, after submitting Msg \#5 (that ends the interaction),

234 we asked them (Final Question) to indicate which of the two versions (the original "Hard" or the

235 colleague suggested "Softer" one), in their opinion, had been actually sent in order to elicit the

236 final answer.

237 The data collection rationale. Our peculiar management of the survey and, specifically, of

238 the participants/survey conductors relationship (SI, Section 0, for details) allow us to exclude that

239 participants' answers are intentionally distorted or insincere. Given this, what data did we exactly

240 collect in our survey? In the first phase (Questions \#1 and \#2) we collected the participants'

241 conscious reports on their interpretations. Naturally, the reports we gathered cannot be

242 considered as reliable descriptions of the "true" interpretation process; rather, they are

243 descriptions of the participants' subjective (conscious) experiences about interpretation. We

244 thought that, even though the link among these conscious accounts and the true process is

245 unknown, the answers could allow us to observe, in a naturalistic-like way, the behaviours

246 associated to the interpretation process. On this basis, we could probably detect enough clues in

247 order to formulate a hypothesis on the deeper "true" process of message interpreting. In other

248 words: we tried an indirect approach given that the interpretation process cannot be directly

249 observed.

250 In the second phase (Questions \#3, \#4 and Final Question), we investigated the

251 relationship between the interpretation of a situation and a consequent decision to be made; such

252 decision was the selection, between the original and the colleague suggested versions of Msg \#4

253 ("Hard" and "Softer" versions), of the one capable to solve the case (i.e. to elicit the final

254 Message \#5). Our thought was that the consistency between interpretation and the following

255 decision could give us either further clues for a deeper understanding of the interpretation

256 process or elements for checking our hypothesis. 
257 Results / 1: Interpretation as a multi-step discontinuous process

258 The results presented in this Section are based on data regarding the first phase of the

259 XX-YY interaction (Messages \#1-3), investigated through the first part of the questionnaire

260 (Questions \#1-2). We recall that each question submitted to the sample sent two inputs: at first,

261 participants were requested to freely interpret some aspects of the messages; then, to account for

262 their own interpretations indicating the "concrete elements" on which these were founded. Given

263 that the two kinds of inputs elicit different kinds of data, we will present separate analyses.

264 Answers to the first input of the questions: the interpretation scatter. The answers to the

265 first input of the questions show that the interpretations provided by participants are widely

266 scattered. Such scatter can be observed for all the messages and for any part of them, even if

267 accurately selected; we have delved further into one of the cases present in our research. Through

268 Question \#2, we firstly asked participants if, comparing Message \#3 with Message \#1, they

269 found the attitude of XX (the sender) towards YY (the receiver) being changed (Method Section

270 and SI, Section 4 for the message texts; SI, Section 4 for the question full-texts). Then, to the 61

271 who answered "YES" (60\% of the sample), we requested to specify how they would define the

272 new XX's attitude. They provided 83 specifications: 64 stated XX's position as strengthened, 12

273 as weakened and 7 unchanged (although these seven, too, had answered "YES" to the first part of

274 Question \#2). In addition, we can find completely opposing statements in these specifications

275 and we can see that scattering covers very different aspects of the XX-YY interaction

276 (behaviours, emotions and so on, Table 5).

277 The observed scatter of interpretations can be represented through a "megaphone-shape"

278 picture (Fig. 1): receivers take into account the same information but their final interpretations 
279 diverge. Such phenomenon is well known, there is plenty of literature about $\mathrm{it}^{12}$. The question is

280 that, even though these observations are common and undisputed, the reasons why this happens

281 remain to be explained.

282 Answers to the second input of the questions: the importance of the not-semantic

283 components. We approached these answers by carefully and sequentially reading them (more

284 than once), and distributing them into homogeneous categories. Such an operation was

285 performed by one of the authors, then discussed and shared with the others; its result consisted in

286 the macro-categories presented in Table 6 . We observed that many of them seemed independent

287 of the message content and of its semantic aspects; in particular, the "Other elements" category

288 contains items totally unrelated to the text semantics and content (a tight selection is presented in

289 Table 7). One of the most interesting indications is the lack of content as a "concrete element"

290 (Table 7, final row): how can an information content express a meaning through its absence?

291 In order to delve further into such matter, we named "components" the categories/sub-

292 categories of the indicated concrete elements and we tried a quantitative analysis. Given that our

293 focus remained on the process, rather than on the sample features, our goal was to provide a

294 rough estimate. Such an estimate was important mainly in relative terms: in case of relative small

295 non-content (non-information) component amounts, we would have to abandon this part of our

296 research. But those amounts were not small. Our analysis of the 1,319 detected components is

297 displayed in Table 8; the indications that clearly focus on the information content constitute only

298 a small minority (around 12\%, see Table 8, “\%” row, "Cont." column) while references to

299 different text components reach, on the whole, about 65\% (Table 8, “\%” row, sum of the first five

300 column totals). The indications referred to some overall effects of the message represent about

${ }^{12}$ About interpretation scatter, we have quoted an example (taken from Hickok, 2009) in our Introduction. In addition, some descriptions, referred to special cases and entailing divergence of interpretations, can be found in Bara \& Tirassa, 1999 (pp. 4-6, communicative meanings as joined constructions); Sclavi, 2003 (pp. 93-98, the "cumulex" play); Campos, 2007 (analysis of a historical communication case). 
$30115 \%$ of the total. About the meaningless components (void of content per se, mere "form"

302 components), their relative amount can be estimated in at least $35 \%$ (holding together symbols,

303 incidental passages, other components and grammatical notations).

304 The proportion of the information content components on the total is very low; even if we

305 sum their relative amount $(12,1 \%)$ to the indications of full sentences or periods $(20,9 \%$, a

306 possible alternative way for referring to the information content) we reach just $1 / 3$ of the total

307 (33\%). The question was important and we carried out a further check: we carefully re-examined

308 the filled questionnaires with reference to the information content component. We found out

309 (Table 9) that one half of the sample (51 people) expresses, among the others, at least 1 reference

310 to such component (no similar hint recordable by the other half). However, only 7 respondents

311 provide a balanced or prevalent amount of indications $(50 \%$, or more, of the individual total)

312 about information content. Among them, only one reaches $100 \%$. Such further observation

313 confirms that references to semantic aspects and information content are a definite minority in

314 participants' indications. We added an ultimate control through checking some statistical

315 distributions related to the components, searching for possible imbalances that could contradict

316 our findings. Nothing emerged (for details see SI, Section 10 and Fig. S4-S7).

317 Following our observations, it seemed that every aspect of even a written message (and

318 even immaterial like an e-mail), regardless of its nature and its intrinsic semantic value, could be

319 treated as a meaningful element of the message, with an extreme degree of scatter among the

320 participants. This was especially surprising because we had used written messages only, bereft of

321 added signals like non-verbal language and context stimuli that usually affect verbal

322 communication (see, for example, Horchak et al., 2014, specially the concept of "situated

323 cognition", and Gibson, Bergen \& Piantadosi, 2013).

324 At this point, we named "disassembling" the observed selective focusing and took two

325 measures. Firstly, we hypothesized a new image for the interpretation process, inverted with 
326 respect to the "megaphone-shape" (Fig. 1) one. Our argument was that, if scatter manifests itself

327 in the beginning (scatter of focus), a "funnel-shape" picture (Fig. 2) could be more suitable:

328 people that select the same component are expected to interpret it in very similar ways. Secondly,

329 we picked up from our data an example of disassembling and decided to carry out an in-depth

330 analysis of it.

$331 \quad$ A disassembling example in detail and a three-step model of the process. Question \#1

332 requested evaluations related to sender-receiver positions and to the relationship between them,

333 on the basis of Messages \#1 and \#2 (see Method Section and SI, Section 4, for the message

334 texts). We found out that 53 people (52\% of the sample) had quoted an expression the sender (the

335 employee "XX", see Method Section and SI, Sections 2, 4) used in Message \#1 ${ }^{13}$ : she premised

336 her request of a technician inspection with the words "we would be pleased if at least once...".

337 This simple expression, apparently trivial, short (8 words in a 67 word message) and in no way

338 highlighted in comparison with the rest of the text, has collected 68 quotations (15 people

339 expressed two, see Footnote 13). Then, respondents have interpreted such specific passage in at

340 least 22 different ways, summarized in Table 10.

341 It seems that focusing on the same component does NOT entail convergent

342 interpretations, that there are TWO levels of scatter instead of one; this could have some

343 important consequences. In terms of metaphors, the previously proposed "funnels" (Fig. 2) were

344 no more suitable; our observations could be much better represented by "hourglasses" (Fig. 3). In

345 terms of process, our observations indicated that the route from the taking into account of a

346 written message (reading it) to the attribution of a conscious meaning to it, could be a sequence

347 of different steps, rather than a unique, homogeneous Input/Output operation (message

${ }^{13}$ The 53 people have reported their interpretations answering Question \#1-a (23), \#1-b (15) or both the questions (15). 
$348 \mathrm{IN} /$ meaning OUT with the brain cortex as "black-box"processor) like it is tacitly assumed in

349 several current approaches.

350 Actually, the two actions of focusing on components and interpreting them seem to have

351 different natures. In order to clear this point, we recall an observation reported in the previous

352 sub-section: on the one hand, respondents explain the conscious meanings they attributed

353 through the outcomes of their individual selective focusing (in their answers, they seem to be

354 literally building-up their meanings on the foundations of the picked-up components). On the

355 other hand, they never explain the reasons why they exactly focused on those components: such

356 focusing manifests "immediately and automatically", priming the attribution of a conscious

357 meaning. In addition, if we would assume that focusing and consciously interpreting have the

358 same nature, our reasoning would fall into an infinite regress ${ }^{14}$. So, we can hypothesize the

359 process of message interpretation like a sequence of different steps: how many steps? We must

360 consider that such process actually starts with the reading of the message; this is just a technical

361 step (learned reading abilities in the used language are required) which turns written signs into

362 words $^{15}$. We named it "decoding" and assumed that its outcomes feed the following step (the

363 selective focusing) whose outcomes, in turn, feed the final one (conscious attribution of meaning,

364 based on rational/logical abilities).

365 In the end, we outlined the model of Fig. 4. The crucial aspect of our hypothesis is the

366 nature of the second step, "disassembling"; on the basis of the presented observations and

367 reflections, we conceive such step as perceptual, not conceptual-logic. The components would

368 act like "physical" stimuli, triggering automatic reactions off ("body" level) in the receivers. We

369 mean: receivers would not consciously recognize the meaning of one component before

$60{ }^{14}$ If the selective focusing on components represents the conscious basis of the attribution of meaning, which

61 could that focusing conscious basis be? And which could be the conscious basis of the conscious basis of that

62 focusing? And so on. A starting point of different nature is anyhow needed.

$63{ }^{15}$ In our opinion, the process should be the same even in case of oral communication (reading and turning written

64 signs into words should just be replaced by listening to and turning spoken sounds into words). 
370 focusing on it; simply, they would focus on those components suitable to trigger their automatic

371 reactions off.

372 One last question remains: if a reader reacts to a given component, even though it appears

373 to be meaningless/contentless, we need to identify what, exactly, that reader perceives. We think

374 we can identify it as the fact that one of these components is present in the message; it can be

375 considered some meta-information to which readers can automatically react (Table 11). This can

376 clarify the aspect of the incidental passage (“...we would be pleased if at least once...”) which

377 triggered the participants' reaction off: the fact that XX had (redundantly) placed it at a certain

378 point of her message ${ }^{16}$.

\section{Results / 2: Unconscious processes in interpretation-action relationship}

380 The results presented in this Section are based on data regarding the second phase of the

381 XX-YY interaction (Message \#4 two versions and Message \#5, see Table 4), investigated through

382 the questions of the questionnaire second part (Questions \#3-4 and Final question). We have

383 submitted to participants two alternative versions of a possible reply to Message \#3: the "Hard"

384 original Message \#4 and the "Softer" colleague suggested version (in short: Msg \#4/H and \#4/S;

385 see Table 4 for the full text messages; SI, Section 5 and Tables S1, S2 for details about the

386 reasons of the proposed alternative). Then, participants were requested to, firstly (Questions \#3

387 and \#4), independently interpret the two versions in terms of their effects on XX; secondly (Final

388 question), to choose the version suitable, in their opinion, to elicit the final XX's answer

389 (Message \#5, that seals the positive ending of the case). Our rationale was the following: the

390 participant's choice could come as a result of the text information conscious processing

${ }^{16}$ It is particularly interesting to note that the expression "the fact that..." is spontaneously used by several respondents in their answers. For example, in the collected questionnaires we can find expressions like the

67 following: "the fact that the arguments are presented through a dotted list"; "the fact that XX is referring to

68 public money”. 
391 (cognitivism stance) or as an automatic reaction independent of every conscious processing

392 (embodied cognition stance). In the first case (our "Hypothesis 0"), the final choices should be

393 outcomes of the interpretations given to the messages; thus, they should result somehow

394 correlated with them. In the second case, no correlation, or a different kind of correlation, should

395 be found (our "Hypothesis 1"). The problem was how to assess such correlation.

$396 \quad$ The coherence between interpretation and choice. Firstly, we displayed (Table 12) the

397 choices indicated by the sample members and found out a strong imbalance between the "Hard"

398 and the "Softer" version of Message \#4. Secondly, we compared the interpretations of Message

399 \#4/H (the "Hard" one) with those of Message \#4/S (the "Softer" one; Table 4 for full-text

400 messages). Source data (opened answers) was purely qualitative. However, answers were easily

401 classifiable into two main categories: predictions for the message inducing a solution of the case

402 (easing or overcoming, anyhow solving the emerging conflict between the interlocutors);

403 predictions for the message inducing a surge, or escalation, in the conflict. We created the

404 dummy variable "Expected effects" and assigned two values to it: "+" in the first condition; "_"

405 in the second one. Finally, we labelled each questionnaire with two new symbols: one referred to

406 the "Hard" Message \#4 (H+ or H-) and one to the "Softer" one (S+ or S-). Methodologically, the

407 labelling has been carried out by one of the authors and, independently, by two external persons.

408 The inter-rater reliability has been checked through Fleiss' kappa and resulted 0,95 (excellent rate 409 of agreement).

410 The combination of the two symbols reports the combined predictions each participant

411 expressed about the effects of the two versions on $\mathrm{XX}: \mathrm{H}+\mathrm{S}+($ both the versions solving the

412 conflict), H+/S- (the "Hard" Message \#4 easing the conflict while the "Softer" Message \#4

413 escalating it), H-/S+ (the opposite), H-/S- (both escalating). Dichotomously displaying "H"

414 against "S" predictions (SI, Section 11a and Table S5) returns a clear convergence on combined

415 prediction "H-/S+"; statistical tests (significance level 5\%) confirm that some correlations 
416 between the interpretations of the "Hard" and the "Softer" version could exist, even though not

417 all cases result significant (Chi-squared test: $p=0.029$, total sample; $p=0.166$, sub-sample

418 “AGE”; $p=0.038$, sub-sample "EMPLOYMENT"; Fischer's Exact test: $p=0.043$, total sample;

$419 p=0.219$, sub-sample “AGE”; $p=0.064$, sub-sample "EMPLOYMENT”). By cross-checking

420 the combined predictions with the final choice (SI, Section 11a and Table S6) we obtained that

421 the most frequent combined prediction (H-/S+) appears to be strongly associated to the "Softer"

422 message choice; indeed, the significance tests show that some further, stronger relations between

423 combined predictions and choice do exist (Chi-squared test: $p=0.001$, total sample; $p=0.035$,

424 sub-sample "AGE”; $p=0.009$, sub-sample "EMPLOYMENT"; Fischer's Exact test: $p=0.002$,

425 total sample; $p=0.027$, sub-sample “AGE"; $p=0.008$, sub-sample "EMPLOYMENT"). Such

426 results led us facing the core-question related to our hypothesis: given the existence of some

427 correlations between choice and combined predictions, which is its direction? We mean: do the

428 interpretations (the predictions) drive the choice (cognitivism stance) or, oppositely, does the

429 choice precede and somehow drive, or overcome, the interpretations (embodied cognition

430 stance)?

431 To delve further into such subject, we created a "coherence indicator" starting from the

432 following premises: (i) The final Message \#5 clearly indicates XX's satisfaction; therefore, the

433 conflict has come to its end. (ii) Now, let us figure a respondent whose answers to Questions \#3

434 and \#4 return a combined prediction H+/S- (the "Hard" Message \#4 solving the conflict, the

435 "Softer" one escalating it). Then, we expect that this respondent indicates the "Hard" Message

436 \#4 in his final choice. Such combination (H+/S- \& "Hard" Msg \#4 choice) would represent the

437 maximum coherence level. (iii) If another respondent provides the same combined prediction but

438 chooses the "Softer" Message \#4 (combination H+/S- \& "Softer" Msg \#4 choice), this would

439 represent the minimum coherence level. (iv) Given the natural variability always recorded in

440 human samples, we expected to find also intermediate coherence levels, based on the other 
441 possible combinations ( $\mathrm{H}+/ \mathrm{S}+$ and $\mathrm{H}-/ \mathrm{S}-)$. These could also be due to the predictable scattering

442 of interpretations about the final Message \#5: someone could interpret it as something different

443 from the sign of the conflict's ending (what happened in a fistful of cases).

444 We defined four coherence levels, increasing from L (low) to LM (low-medium), MG

445 (medium-great) and G (great); the scale is fully represented in SI, Section 11a and Table S7. This

446 way, it has been possible to study the sample distribution with respect to coherence levels (Table

447 13). The histogram for the whole sample (Figure 5, data from Table 13) shows the expected

448 shape except for the frequency of the low coherence bin, over-represented. Actually, we expected

449 L frequency to be null or very close to null; anyway, it should result the lowest of all. On the

450 contrary, we found L values higher than the LM ones, representing $12.2 \%$ of the sample. The two

451 control sub-samples (right columns of Table 13) show fully comparable features.

452 At this point, we refined our analysis displaying separately distributions of "H" and "S"

453 choosers; for the reliability of comparison, we excluded data referred to the respondents having

454 just primary education levels (only 4 out of 102 in our sample). Data is displayed in Table 14 and

455 complemented in SI, Section 11b, Tables S8, S9; all the Tables show a surprising asymmetry

456 whose significance is confirmed by Chi-squared test and Fisher's Exact test (in all cases

$457 \mathrm{p}<0.001)$. Graphic representations render even better such asymmetry: the total sample

458 histograms (Fig. 6, percent distributions from Table 14) show that the percent frequency of the

459 "Softer" message choosers (white bins) increases regularly from L category to G, reminding (as

460 expected) of certain power, or exponential, curves. Oppositely, the percent frequency of the

461 "Hard" message choosers (grey bins) is arranged in an irregular, almost bimodal shape. We

462 checked these distribution shapes by using many different sub-samples (selection displayed in SI,

463 Section 11b, Fig. S8-S11), included the already mentioned "Age" (Fig. 7, data from SI, Section

464 11b, Table S8) and "Employment" (Fig. 8, data from SI, Section 11b, Table S9) sub-samples. We

465 always obtained the same significant imbalance. 
467 correlation between the participants' choice and the coherence level; but what about its strength

468 and its direction? In order to investigate the strength, we calculated the odds ratio. Our success

469 item was the L level, our failure items were all the other coherence levels. Using data from Table

47014 , we can find ODDS1 $=0.417$ (the "Hard" version choosers, about 1 success for each failure)

471 and ODDS2 $=0.028$ (the "Softer" version choosers, 1 success every about 36 failures). The final

472 result is ODDS RATIO $=25.5$ which highlights a strong correlation between the " $\mathrm{H}$ " choice and

473 the L coherence level. As much as to say that, if you choose the "Hard" version of message \#4, it

474 is much more likely (with respect to the "Softer" version choosers) that your choice is

475 inconsistent with your interpretations of the two messages. About the direction of such

476 correlation (the interpretations precede and drive the choice or the choice is independent of

477 interpretations), we think the first s is not tenable; indeed, it could be confirmed just in case of

478 general consistency between interpretations and choice.

All this contrasts our "hypothesis 0": the participants' choice does not seem to come as a result of the text information conscious processing. Then, the choice should be independent of the previous interpretations, what upholds our "hypothesis 1". After this first conclusion, we set

482 up a second indicator ("block preference" indicator) to further check our hypothesis. For text 483 length reasons, we present details about such indicator, its employment, and relative analysis in

SI, Section 12 with Tables S10-S13. We found no contradictions with the previous results.

\section{Discussion}

486 With regards to method, our work showed that studying the interpretation of natural

487 language messages in natural-like conditions can complement laboratory studies based on

488 isolated words/phrases and contribute to a wider comprehension of the phenomenon. With 489 regards to results, the picture outlined through the first part of our work can be synthesized as 
490 follows: (i) The interpretation process begins with an operation that looks like a selective and

491 subjective picking up of (or focusing on) the most different components, rather than being a

492 systematic, conscious scanning of the text content. Such behaviour is widely scattered: in the

493 whole research, with regards to each specific message, it is impossible to find two identical

494 combinations of components in participants' answers; (ii) Readers seem to make no distinction

495 among intrinsically meaningful or meaningless components: the meaning they attribute can

496 derive from any "chunk" of the text or from any other text or non-text element arbitrarily chosen;

497 (iii) While the final meaning attributed to the message is justified through the indicated

498 components, no reason (at all, in any cases) is provided for that selection: in the participants'

499 answers, the focused components suddenly appear; they are presented just as "given", and

500 without any doubt ${ }^{17}$. On these bases, we have proposed a three-step model for the interpretation

501 process (Fig. 4); the crucial step is the second one ("disassembling") which, in our hypothesis, is

502 an automatic reaction, out of conscious control. It precedes and feeds forward the conscious

503 attribution of meaning to the message ${ }^{18}$.

504 If our hypothesis will be confirmed, this means that words are not mere symbols; they are

505 also stimuli (they can act like physical stimuli) that trigger automatic reactions off in the

506 receivers $^{19}$. It also means that the third step (conscious attribution of meaning) is fed by the

507 outcomes of the unconscious reaction (“disassembling”), rather than by the original message;

${ }^{17}$ The unique doubt expressed in the whole research is the following: 1 participant (out of 102) declares uncertainties in his final choice (between the "Hard" version of Msg \#4 and the "Softer" one) writing that the final effect could be obtained with both the messages. It must be noted that, with regards to the other questions, this special participant's answers are totally doubt-free.

${ }^{18}$ We have noted that, if disassembling were a conscious passage having the same nature of the following conscious attribution of meaning, the analysis would turn into an infinite regress (see Footnote 14).

${ }^{19}$ Such ambivalence looks interestingly (or just curiously?) similar to what happens in certain physics phenomena like the double nature of light (waves/particles) or the uncertainty about some features of many atomic particles. In those cases, the ambivalence is solved just in the process of measuring the phenomena (Zeilinger, 2010, for a discussion about the case of photons, and von Baeyer, 2013 for a recent point of view about such ambivalence); in the case of words, something similar would happen, given that their nature would become evident just in relation with the receiver's reaction. 
508 our conscious direct contact with the real world would be prevented, and we would actually

509 attribute conscious meanings just to our automatic reactions to it. In short: through the first part

510 of our work, we have outlined the possible structure of the message interpretation process.

511 The second part of our work has been designed in a way similar to a social psychology

512 experiment; through it, we have worked downstream with respect to the interpretation process

513 itself, investigating its effects on a consequent behaviour (the final choice); we found out

514 significant imbalances in the coherence between interpretation and choice. Roughly, we can label

515 "rational" the choices that show maximum coherence with the previous interpretations of the two

516 messages (the original "Hard" Message \#4, and the suggested "Softer" version); conversely, we

517 can label "irrational" the choices that show minimum coherence. We found that the irrational

518 cases are significantly ascribable to " $\mathrm{H}$ " version choosers rather than to "S" version choosers. In

519 other words: the elements provided by interpretations appear insufficient to determine the choice;

520 this means that other factors intervene. Such factors should be unconscious, otherwise they

521 would be declared by at least some participants; in addition, they must have a different and

522 stronger source with regards to the conscious/rational analysis of the message content, otherwise

523 their influence on the choice would not prevail.

524 The main question is: why, in the decision process, do these factors significantly weigh

525 just in connection with one choice and not with the other one? Further research is needed to find

526 the answer. Provisionally, we think there are two possible hypotheses: (i) The two sub-samples

527 follow different paths in interpreting natural language messages ("Softer" version choosers

528 would base their choices on rational information processing, which would precede action, while

529 "Hard" version choosers would react instinctively and choose before analysing the available

530 information); (ii) The two sub-samples actually follow the same path (automatic reaction

531 preceding conscious information processing, in our opinion) and the difference they show is

532 linked to the differences in their automatic reaction schemes ("Softer" version choosers' reaction 
533 would privilege the attention to the relational aspects while "Hard" version choosers' reaction

534 would privilege the content aspects).

535 Situating our results in the current research scenario. With respect to the dispute between

536 the stance of cognitivism and the embodied cognition hypotheses, we think our research could be

537 situated in a third position, for two reasons. The first reason is that, while these theories share

538 (even though they come to opposite conclusions) the concept of natural language interpretation

539 as a unique operation, we have seen it as a discontinuous process (three steps of different nature).

540 The second reason is that, in our model, two of the three sub-processes seem to be compatible,

541 separately, with those two theories. We mean: the embodied concept features are akin to our

542 second step ("disassembling"); the cognitivist hypothesis is clearly akin to our third step, (see

543 Fig. 4).

544 Probably, we can better exemplify this through recovering the example (see Hickcok,

5452009 , page 1240) we presented in the Introduction. In our opinion, embodied cognition

546 hypothesis looks at that described act of pouring in its purely motorial nature; conversely,

547 understanding it, for example, as "pouring" or "filling", requires the interpretation of a situation

548 which is not limited to the act per se. In order to attribute the "pouring" meaning, one must focus

549 on the liquid flow direction (inside to outside the bottle); for the "filling" meaning, one must

550 focus on the glass receiving the liquid; for the "emptying" meaning, one must focus on the

551 amount of liquid inside the bottle. The attribution of conscious meanings should be preceded by

552 the previous, unconscious selection of specific points of view (something closely resembling our

553 “disassembling” step).

554 Apart from this, if we extend back our literature survey, we can find, for example, that 555 conscious thinking following (rather than preceding) "body" reactions can be traced back up to

556 the hypotheses of the Nineteenth Century philosopher and psychologist William James. In one of 557 his examples (the "James's bear", see James, 1890, Chapter XXV), James explains his theory of 
558 emotions suggesting that, for example (our synthesis), we do not run away from a bear because

559 we see it, we know it is very dangerous, so we are scared of it and, consequently, we consciously

560 decide to run away (as common sense would sustain). Conversely, we feel we are afraid because

561 (consciously and successively) we discover our body having started a desperate run. In other

562 words: what we call "emotion" is usually intended as a body reaction consequent to the rational

563 processing of consciously perceived environmental stimuli; James suggests that the body

564 reaction immediately follows perception and what we call "emotion" is the consciousness of the

565 new body state (a form of self-consciousness). We are aware that James theory (exactly: James-

566 Lange theory) has been criticized and that alternative theories have been proposed (for example

567 Cannon, 1927; Schachter \& Singer, 1962); nevertheless, we do refer to it because recent

568 scientific research and reviews seem to suggest some re-consideration of the matter (for example,

569 Friedman, 2010).

570 In the Twentieth Century, we can find the Gregory Bateson's approach to human

571 communication conceived as a system and to the question of the receiver's active role; he uses a

572 strictly formal presentation (see Bateson, 1972, in particular Chapter 4.8 on the logical categories

573 of communication, founded on Russel and Whitehead's theory of logical types). In addition, we

574 recall a group of theories and models (some of which expressly refer to Bateson's studies) that

575 tackle the question mainly from a pragmatic slant: the so called "pragmatic models" (Berne,

576 1961; Watzlawick, Beavin Bavelas \& Jackson, 1967; Bandler \& Grinder, 1975). Conceived

577 inside a psychoanalytic context, they all put perception and stimuli at the centre of their attention

578 and reverse the relationship between action and thought using action (rather than thought) to

579 induce training and therapeutic effects ${ }^{20}$. We find no important contradictions among our

$81{ }^{20}$ On the one hand, it is worth mentioning a special work coming from NLP founders (Grinder \& Bandler,

82 1979): it appears different from the work that founded this theory (Bandler \& Grinder, 1975) and that has

83 successively been developed by NLP specialists (for example Dilts, 1998). As a matter of fact, that work

84 gives a central role to perception and to physical stimuli (not mediated by language) as a possible

85 communication and therapeutic instrument (see, in particular, the concept of "sensorial anchors" in Grinder \&

86 Bandler, 1979). On the other hand, we should remind a Watzlawick's work on the modern evolution of 
580 hypotheses and such models; rather, we find complementarity: they show how physical stimuli

581 can act like messages; our results could show that words (even if only written) can act like

582 physical stimuli.

583 About the relevance of unconscious processes in human behaviour, some fundamental

584 clarification is provided by Custers \& Aarts, 2010 through a review of experimental works; it re-

585 examines the disputed question of the passage from perception to action. The authors compare

586 the traditional positions of Sensory-motor Principle (SMP, for example Massaro \& Cowan, 1993;

587 for a presentation and discussion about the sequential processing of stimuli conceived as the

588 foundation of human/environment interactions, see also Rizzolatti \& Sinigaglia, 2006, chapters

589 1, 2) and Ideomotor Principle (IMP, Stöcker \& Hoffmann, 2004; Pezzulo et al., 2006; Melcher et

590 al., 2008; for a synthesis, Iacoboni, 2008, Chapter 2, pp. 56-57 of Italian edition). Doing so, they

591 show how certain stimuli (images, solid objects or even written words), intentionally added to an

592 experimental setting, can alter the sample behaviours, even if such stimuli are not consciously

593 detected: "under certain conditions, actions are initiated even though we are unconscious of the

594 goals to attain... [and] goal pursuit can... operate unconsciously" (Custers \& Aarts, 2010). They

595 also sustain that arguments frequently presented as rational motivations for action are, actually,

596 ex-post justifications of unconsciously performed behaviours.

597 The role of physical stimuli in swaying communication through natural language is

598 confirmed by a series of recent works (for example Zhong, Bohns \& Gino, 2010; Tsay, 2013;

599 and, for a popular-scientific coverage, Lobel, 2014). Further, quite unpredictable factors that can

600 sway message interpretation can be the specific national languages used (for example Marian \&

601 Kaushanskaya, 2005; Costa et al., 2014) or the metaphors used to express concepts (Thibodeau 
602 \& Boroditsky, 2011; Thibodeau \& Boroditsky, 2013). Our data is consistent with the outlined

603 scenario in that it confirms the effects of perception-reaction on conscious processing.

$604 \quad$ Some possible consequences. Naturally, our results need to be confirmed; once they

605 would be, we can see four main possible consequences. The first one concerns the discontinuous

606 nature of the interpretation process and, specifically, the role of the second step of our model

607 (disassembling) in human communication through natural language: some traditional empirical

608 knowledge would find theoretical bases (for example in advertising and marketing fields) and a

609 revision of human communication current models would be needed (for example with regards to

610 mass media and education). Simply, the fact should be taken into account that human

611 communication through natural language could work in a slightly different way than expected

612 and thought up until now.

613 The second consequence would be the analogical, rather than digital, basis of

614 interpretation. Meaning would be established starting from the body automatic reaction in the

615 “disassembling step", analogically triggered through individual reaction schemes. This could

616 lead to consider natural language expertise as a system of acquired reflexes, what would mean

617 that human beings would "communicate through their body" in a wider and deeper sense than

618 conceived at present (something quite different from mere non-verbal language performances).

619 Such feature could heavily affect the possibility to reproduce human interpretation process on

620 digital computers, regardless of their processing power and data storage capacity. The two

621 systems could result incompatible, rather than simply different. We are not the first who propose

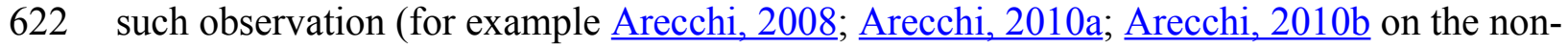

623 algorithmic nature of knowledge and intelligence; Arecchi, 2010d on creativity as NON-bayesian

624 process). In such perspective, if there is any possibility to reproduce the human interpretation

625 process on a computational device, then its model should be the whole human being, not the sole

626 brain cortex. Consequently, what really can prevent present times computers from imitating 
627 human thought is not insufficient data processing power or data storage capacity; rather, it is the

628 lack of a special peripheral unit: a human body.

629 The third consequence could derive from our observations about the taking into account

630 of the message components by the reader, that seems to be performed like a subjective operation,

631 quite arbitrary and unpredictable. If this will be confirmed, the concept of "content of a message"

632 should probably be revised, given that it would result impossible to ex-ante define all the

633 contents a reader could detect in a specific message. What is more, as a fourth possible

634 consequence, if mere "form" (aesthetic) components are indifferently taken into account as

635 sources of meaning with respect to the content components, then the difference between form

636 and content fades, leading to a concept of "message" as a unit made up only by components, all

637 of them having the same importance (the same ex-ante probability of being chosen).

$638 \quad$ Conclusion

639 At the end of our arguing about the attribution of meaning, it is worth briefly considering 640 the problem of "what is meaning" (what is the meaning of "meaning"). Beyond the strictly

641 phylosophical, abstract definitions, nowadays we can record attempts to provide operative

642 definitions; for example Guastello, 2002, who considers the sender-receiver couple as a complex

643 system and the meaning like an emergent phenomenon which characterizes it. Our research can

644 lead us to hypothesize another operative definition of "meaning": The meaning attributed to a

645 message is the receiver's synthetic conscious report (through natural language) on the final state

646 of his/her organism after experiencing the interaction with the message.

647

648 Acknowledgements 
649 We thank Laura Baglietto, Andrea Baldini, Marco Calabrò, Leonardo Cavari, Hasna El-Hachimi, 650 Alessandro Farini, Alessandra Gasperini, Maddalena Morandi, Claudia Santovito, Arabella

651 Tanyel-Kung for their comments.

652 A special thanks to Fortunato Tito Arecchi for his suggestions; to Andrea Fiaschi and Christina

653 Tsirmpa for their text revision; to Letizia Scrobogna for her contribution to data revision; to Irene 654 Maffei for her final text survey and impact assessment. 


\section{References}

656 Arecchi FT. 2008. Coerenza, Complessità, Creatività. Roma: Di Renzo.

657 Arecchi FT. 2010a. Coherence, complexity and creativity: from lasers to cognitive processes.

658 Giornale di Fisica - Quaderni di Storia della Fisica 16 (2010):157-183.

659 Arecchi FT. 2010b. Coherence,complexity and creativity: the dynamics of decision making. In:

660 Faggini M, Vinci CP, ed. Decision theory and choices: a complexity approach. Milan: Springer-

661 Verlag Italia, 3-21.

662 Arecchi FT. 2010c. Dynamics of consciousness: complexity and creativity. The Journal of 663 Psychophysiology (2010) 24 (2): 141-148.

664 Arecchi FT. 2010d. The physics of mental acts: coherence and creativity. Journal of Physics:

665 Conference Series 174, 012010 (2009).

666 Arecchi FT. 2011. Chaos and Complexity. In: Jencks C ed. The Post-Modern Reader. Chichester: 667 John Wiley \& Sons, 279-283.

668 Arévalo A, Perani D, Cappa SF, Butler A, Bates E, Dronkers N. 2007. Action and object

669 processing in aphasia: From nouns and verbs to the effect of manipulability. Brain and Language

670100 (2007) 79-94. DOI : 10.1016/j.bandl.2006.06.012.

671 Aziz-Zadeh L, Damasio A. 2008. Embodied semantics for actions: Findings from functional brain

672 imaging. Journal of Physiology - Paris. DOI: 10.1016/j.jphysparis.2008.03.012. 
673 Aziz-Zadeh L, Wilson SM, Rizzolatti G, Iacoboni M. 2006. Congruent embodied representations

674 for visually presented actions and linguistic phrases describing actions. Current Biology 16

675 (September): 1818-1823. DOI: 10.1016/j.cub.2006.07.060.

676 Bandler R, Grinder J. 1981. La struttura della magia. Roma: Astrolabio-Ubaldini. [Or. ed.:

677 Bandler R, Grinder J. 1975. The structure of magic. Palo Alto: Science \& Behaviour Books.]

678 Bara BG, Tirassa M. 1999. A mentalist framework for linguistic and extralinguistic

679 communication. In: Bagnara S, ed. Proceedings of the 3rd European Conference on Cognitive

680 Science (ECCS '99). Roma: Istituto di Psicologia del CNR.

681 Barthes R. 2000. La retorica antica. Milano: Bompiani. [Or. ed.: Barthes R. 1970. L'ancienne

682 rhétorique. Communications 16: 172-223. DOI: 10.3406/comm.1970.1236.]

683 Bateson G. 1976. Verso un'ecologia della mente. Milano: Adelphi. [Orig. ed.: Bateson G. 1987

684 (1972). Steps to an Ecology of Mind: Collected Essays in Anthropology, Psychiatry, Evolution, 685 and Epistemology. Northvale: Aronson.]

686 Bedny M, Caramazza A, Grossman E, Pascual-Leone A, Saxe R. 2008. Concepts are more than 687 percepts: The case of action verbs. The Journal of Neuroscience, October 29, 2008, 688 28(44):11347-11353. DOI: 10.1523/JNEUROSCI.3039-08.2008.

689 Bedny M, Caramazza A, Pascual-Leone A, Saxe R. 2012. Typical Neural Representations of

690 Action Verbs Develop without Vision. Cerebral Cortex February 2012, 22: 286-293. DOI:

691 10.1093/cercor/bhr081. 
692 Bedny M, Caramazza A. 2011. Perception, action, and word meanings in the human brain: the 693 case from action verbs. Annals of the New York Academy of Sciences 1224: 81-95. DOI:

694 10.1111/j.1749-6632.2011.06013.x.

695 Berne E. 1971. Analisi transazionale e psicoterapia. Roma: Astrolabio-Ubaldini. [Or. ed.: Berne 696 E. 1961. Transactional analysis. New York: Grove.]

697 Bobrowski O, Meir R, Eldar YC. 2009. Bayesian filtering in spiking neural networks: noise, 698 adaptation, and multisensory integration. Neural Computation 2009 May, 21(5): 1277-320.

699 Campos MN. 2007. Ecology of meanings: A critical constructivist communication model. 700 Communication Theory 17: 386-410. DOI: 10.1111/j.1468-2885.2007.00304.x.

701 Cannon WB. 1927. The James-Lange theory of emotions: a critical examination and an 702 alternative theory. The American Journal of Psychology Vol 39, 1927, 106-124. DOI: $70310.2307 / 1415404$

704 Chater N, Tenenbaum JB, Yuille A. 2006. Probabilistic models of cognition: Conceptual 705 foundations. TRENDS in Cognitive Sciences Vol.10, No.7, July 2006.

706 Christian B. 2012. Essere umani: che cosa ci dice di noi il test di Turing. [Or. ed.: Christian B.

707 2011. The most human human: What talking with computers teaches us about what it means to 708 be alive. New York-London: Doubleday - Random House.]

709 Costa A, Foucart A, Hayakawa S, Aparici M, Apesteguia J, Heafner J, Keysar B. 2014. Your 710 Morals Depend on Language. PLoS ONE 9(4): e94842. DOI: 10.1371/journal.pone.0094842. 
711 Crepaldi D, Aggujaro S, Arduino LS, Zonca G, Ghirardi G, Inzaghi MG, Colombo M, Chierchia

712 G, Luzzatti C. 2006. Noun-verb dissociation in aphasia: the role of imageability and functional

713 locus of the lesion. Neuropsychologia 2006; 44 (1): 73-89. PMID: 15922372.

714 Custers R, Aarts H. 2010. The unconscious will. Science 329: 47-50. DOI:

$71510.1126 /$ science. 1188595.

716 De Mauro T. 2003 (1980). Guida all'uso delle parole. Roma: Editori Riuniti.

717 di Pellegrino G, Fadiga L, Fogassi L, Gallese V, Rizzolatti G.1992. Understanding motor events:

718 A neurophysiological study. Experimental brain research 91: 176-180. DOI :

$71910.1007 / \mathrm{BF} 00230027$.

720 Dilts R. 2003. Creare modelli con la PNL. Roma: Astrolabio - Ubaldini. [Or. ed.: Dilts R. 1998.

721 Modeling with NLP. Capitola: Meta Publications.]

722 Ferrari F, Rizzolatti G. 2014. Mirror neurons research: the past and the future. Phil. Trans. R.

723 Soc. B 369, 20130169 (published 28 April 2014). DOI: 10.1098/rstb.2013.0169.

724 Ferri F, Riggio L, Gallese V, Costantini M. 2011. Objects and their nouns in peripersonal space.

725 Neuropsychologia 49 (2011): 3519- 3524.

726 Fowler CA, Galantucci B, Saltzman E. 2003. Motor theories of perception. In: Arbib MA, ed.

727 The handbook of brain theory and neural networks. Cambridge: The MIT Press, $2^{\mathrm{d}}$ edition, 705728707.

729 Fox C, Stafford T. 2012. Maximum utility unitary coherent perception vs. the Bayesian brain.

730 Proceedings of the Annual Meeting of the Cognitive Science Society, Sapporo 1-4 August 2012. 
731 Available: http://mindmodeling.org/cogsci2012/papers/0070/paper0070.pdf(accessed 1 June

732 2015).

733 Friedman BH. 2010. Feelings and the body: the Jamesian perspective on autonomic specificity of

734 emotion. Biological Psychology, Jul;84(3):383-93. DOI: 10.1016/j.biopsycho.2009.10.006. Epub

7352009 Oct 29.

736 Gallese V, Gernsbacher MA, Heyes C, Hickok G, Iacoboni M. 2011. Mirror Neurons Forum.

737 Perspectives on Psychological Science 2011 6: 369. DOI: 10.1177/1745691611413392.

738 Gallese V, Rochat M, Cossu G, Sinigaglia C. 2009. Motor cognition and its role in the phylogeny

739 and ontogeny of intentional understanding. Developmental Psychology 45: 103-113.

740 Gallese V, Sinigaglia C. 2011a. How the body in action shapes the self. Journal of Consciousness

741 Studies 18: No. 7-8, 117-43.

742 Gallese V, Sinigaglia C. 2011b. What is so special about embodied simulation? Trends in

743 cognitive neuroscience 2011 Nov, 15(11): 512-9.

744 Gallese V, Sinigaglia C. 2012. Response to de Bruin and Gallagher: Embodied simulation as

745 reuse is a productive explanation of a basic form of mind-reading. Trends in Cognitive Sciences

746 February 2012, Vol. 16, No 2: 99-100. DOI: 10.1016/j.tics.2011.12.002.

747 Gallese V. 2000. The inner sense of action. Journal of Consciousness studies 7, 10: 23-40.

748 Gallese V. 2005. Embodied simulation: From neurons to phenomenal experience.

749 Phenomenology and the Cognitive Sciences 4: 23-48. 
750 Gallese V. 2006. Intentional attunement: A neurophysiological perspective on social cognition

751 and its disruption in autism. Brain Research 1079: 15-24.

752 Gallese V. 2007. Before and below “theory of mind": Embodied simulation and the neural

753 correlates of social cognition. Philosophical Transactions of the Royal Society B: Biological

754 Sciences 362: 659-669.

755 Gallese V. 2008 Mirror neurons and the social nature of language: The neural exploitation 756 hypothesis. Social Neuroscience 3: 317-333.

757 Gallese V. 2009a. Motor abstraction: A neuroscientific account of how action goals and intentions 758 are mapped and understood. Psychological Research 73: 486-498.

759 Gallese V. 2009b. Neuroscienze controverse: il caso dei neuroni specchio. Interview by Marco

760 Mozzoni. Brainfactor 29/5/2009. Available: http://www.brainfactor.it/?p=1254 (accessed 1 June 761 2015).

762 Gallese V. 2010. The Mirror Neuron Mechanism and Literary Studies. Interview by Hannah

763 Chapelle Wojciehowski. University of California eScholarship 2010. Available:

764 http://escholarship.org/uc/item/56f8v9bv (accessed 1 June 2015).

765 Gallese V. 2011. Embodied Simulation Theory: Imagination and Narrative. Neuropsychoanalysis $7662011,13(2)$.

767 Gallese V.2014. Bodily selves in relation: embodied simulation as second-person perspective on 768 intersubjectivity. Phil. Trans. R. Soc. B 369, 20130177 (published 28 April 2014). DOI:

$76910.1098 /$ rstb.2013.0177. 
770 Geymonat L. 1970. Storia del pensiero filosofico e scientifico. Milano: Garzanti, Vol. 1, Sez. I.

771 Gibson E, Bergen L, Piantadosi ST. 2013. Rational integration of noisy evidence and prior

772 semantic expectations in sentence interpretations. Proceedings of the National Academy of

773 Sciences May 14, 2013, Vol. 110, n. 20: 8051-8056.

774 Goldman A, de Vignemont F. 2009. Is social cognition embodied? Trend in cognitive sciences

775 April 2009, 13(4): 154-9.

776 Goldstein R. 2006. Incompletezza: La dimostrazione e il paradosso di Kurt Gödel. Torino:

777 Codice Edizioni. [Or. ed.: Goldstein R. 2005. Incompleteness: The proof and paradox of Kurt

778 Gödel. New York-London: Norton.]

779 Griffiths TL, Kemp C, Tenenbaum JB. 2008. Bayesian models of cognition. In: Ron Sun, ed.

780 Cambridge Handbook of Computational Cognitive Modelling. Cambridge: Cambridge University

781 Press.

782 Grinder J, Bandler R. 1980. La metamorfosi terapeutica: Principi di Programmazione

783 Neurolinguistica. Roma: Astrolabio-Ubaldini. [Or. ed.: Grinder J, Bandler R. 1979. Frogs into

784 princes: Neuro Linguistic Programming. Moab: Real People Press.]

785 Guan CQ, Meng W, Yao R, Glenberg AM. 2013. The Motor System Contributes to

786 Comprehension of Abstract Language. PLoS ONE 8(9): e75183. DOI:

787 10.1371/journal.pone.0075183.

788 Guastello SJ. 2013 (2002). Managing emergent phenomena: nonlinear dynamics in work

789 organizations. Abingdon (UK): Taylor \& Francis, Psychology Press. 
790 Hickok G. 2009. Eight problems for the mirror neurons theory of action understanding in

791 monkeys and humans. Journal of Cognitive Neuroscience 21:7: 1229-1243. DOI:

$79210.1162 /$ jocn.2009.21189.

793 Hoffman P, Lambon Ralph MA. 2011. Reverse concreteness effects are not a typical feature of

794 semantic dementia: Evidence for the hub-and-spoke model of conceptual representation.

795 Cerebral Cortex, September 2011;21:2103-2112. DOI: 10.1093/cercor/bhq288

796 Horchak OV, Giger JC, Cabral M, Pochwatko G. 2014. From demonstration to theory in

797 embodied language comprehension: a review. Cognitive Systems Research Volumes 29-30,

798 September 2014: 66-85. DOI: 10.1016/j.cogsys.2013.09.002.

799 Iacoboni M, Molnar-Szakacs I, Gallese V, Buccino G, Mazziotta JC, Rizzolatti G. 2005. Grasping

800 the intentions of others with one's own mirror neuron system. PLoS Biology 3(3): e79.

801 Iacoboni M. 2008. I neuroni specchio: Come capiamo ciò che fanno gli altri. Torino: Bollati

802 Boringhieri. [Or. ed.: Iacoboni M. 2008. Mirroring People: The New Science of How We Connect

803 with Others. New York: Farrar, Straus \& Giroux.]

804 Ingram JN, Körding KP, Howard IS, Woolpert DM. 2008. The statistics of natural hand

805 movements. Experimental Brain Research 188: 223-236. DOI 10.1007/s00221-008-1355-3.

806 James W. 1890. The principles of psychology. New York: Holt.

807 Jeannerod M, Arbib MA, Rizzolatti G, Sakata H. 1995. Grasping objects: Cortical mechanisms of

808 visuomotor transformation. Trends in Neuroscience 18: 314-320. 
809 Khosravi H, Bina B. 2010. A survey on statistical relational learning. Lecture Notes in Computer

810 Science, Volume 6085/2010: 256-268. DOI: 10.1007/978-3-642-13059-5_25.

811 Krauss RM, Fussell SR. 1996. Social psychological models of interpersonal communication. In:

812 Higgins ET, Kruglanski A, ed. Social Psychology: A handbook of basic principles. New York:

813 Guilford, 655-701.

814 Leavitt D. 2007. L'uomo che sapeva troppo: Alan Turing e l'invenzione del computer. Torino:

815 Codice Edizioni. [Or. ed.: Leavitt D. 2006. The man who knew too much: Alan Turing and the

816 invention of the computer. New York-London: Norton.]

817 Liberman AM, Whalen DH. 2000. On the relation of speech to language. Trends in Cognitive

818 Neuroscience 4: 187-196.

819 Lobel T.2014. Sensation: the new science of physical intelligence. New York: Simon \&

820 Schuster/Atria Books.

821 Locke JL. 2009. Evolutionary developmental linguistics: Naturalization of the faculty of

822 language. Language Sciences 31 (2009) 33-59. DOI: 10.1016/j.langsci.2007.09.008.

823 Lotze RH. 1852. Medicinische psychologie oder physiologie der seele. Leipzig: Weidmannsche 824 Buchandlung.

825 Mach E.1897. Contributions to the analysis of the sensations. Chicago: Open Court.

826 Maffei R, Cavari L, Ranieri M. 2007. L'autre face du changement: Constants et structures dans la

827 collaboration en ligne. Proceedings of the Colloques TICE - Méditerranée 2007. ISDM 29.

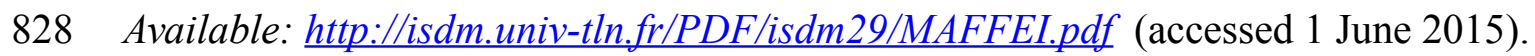


829 Maffei R. 2006. Questioni di stile: L'influenza dello stile di conduzione sui gruppi collaborativi

830 online. Proceedings of the Colloques TICE - Méditerranée 2006. ISDM 25. Available:

831 http://isdm.univ-tln.fr/PDF/isdm25/Maffei_TICE2006.pdf (accessed 1 June 2015).

832 Mahon B Z, Caramazza A. 2011. What drives the organization of object knowledge in the brain?

833 Trends in Cognitive Sciences, Volume 15, Issue 3, 97-103, 14. DOI : 10.1016/j.tics.2011.01.004.

834 Mahon BZ, Caramazza A. 2008. A critical look at the Embodied Cognition Hypothesis and a new 835 proposal for grounding conceptual content. Journal of Physiology - Paris 102: 59-70.

836 DOI:10.1016/j.jphysparis.2008.03.004.

837 Mahon BZ, Caramazza A. 2009. Concepts and categories: A cognitive neuropsychological

838 perspective. Annual Review of Psychology 60: 27-51. DOI:

839 10.1146/annurev.psych.60.110707.163532.

840 Marcus G. 2004. La nascita della mente: Come un piccolo numero di geni crea la complessità

841 del pensiero umano. Torino: Codice Edizioni. [Or. ed.: Marcus G. 2004. The birth of the mind:

842 How a tiny number of genes creates the complexities of human thought. New York: Basic Books.]

843 Marian V, Kaushanskaya M. 2005. Autobiographical memory and language in bicultural

844 bilinguals. In: Cohen J, McAlister KT, Rolstad K, MacSwan J, eds. Proceedings of the $4^{\text {th }}$

845 International Symposium on Bilingualism. Somerville (MA): Cascadilla Press.

846 Marino BFM, Gough P, Gallese V, Riggio L, Buccino G. 2011. How the motor system handles

847 nouns: A behavioural study. Psychological Research (published online). DOI 10.1007/s00426-

$848 \quad 011-0371-2$ 
849 Massaro DW, Cowan N. 1993. Information Processing Models: Microscopes of the mind. Annual

850 Review of Psychology 44: 383-425.

851 Melcher T, Weidema M, Eenshuistra RM, Hommel B, Gruber O. 2008. The neural substrate of

852 the ideomotor principle: An event-related fMRI analysis. NeuroImage 39: 1274-1288. DOI:

853 10.1016/j.neuroimage.2007.09.049.

854 Menchetti S, Costa F, Frasconi P, Pontil M. 2005. Wide coverage natural language processing

855 using kernel methods and neural networks for structured data. Science Direct, Pattern

856 Recognition Letters 26 (2005): 1896-1906. Available:

857 http://www.researchgate.net/publication/222681214 Wide coverage natural language processi

858 ng using kernel methods and neural networks for structured data (accessed 1 June 2015).

859 Merleau-Ponty M. 1965. La fenomenologia della percezione. Milano: Il Saggiatore. [English ed.:

860 Merleau-Ponty M. 1962. Phenomenology of perception. London-New York: Routledge.]

861 Mitchell TM. 1997. Machine learning. New York: McGraw Hill.

862 Mitchell TM. 2009. Brains, meaning and corpus statistics. Google Tech Talks March 27, 2009.

863 Available: http://www.youtube.com/watch?v=QbTf2nE3Lbw (accessed 1 June 2015).

864 Moseley RL, Pulvermüller F. 2014. Nouns, verbs, objects, actions, and abstractions: Local fMRI

865 activity indexes semantics, not lexical categories. Brain and language 132 (2014): 28-42. DOI:

866 10.1016/j.bandl.2014.03.001.

867 Nardone G, Watzlawick P. 1990. L'arte del cambiamento. Milano: Ponte alle Grazie. 
868 Negri GAL, Rumiati RI, Zadini A, Ukmar M, Mahon BZ, Caramazza A. 2007. What is the role of

869 motor simulation in action and object recognition? Evidence from apraxia. Cognitive

870 Neuropsychology 24(8): 795-816. DOI: 10.1080/02643290701707412.

871 Newell A, Shaw JC, Simon HA. 1958. Elements of a theory of human problem solving.

872 Psychological Review 65: 151-166. DOI 10.1037/h0048495.

873 Pascolo PB, Budai R. 2013. Just how consistent is the mirror neuron system paradigm? Progress

874 in Neuroscience 2013; 1 (1-4): 29-43. DOI: 10.14588/PiN.2013.Pascolo.29

875 Patterson K, Nestor PJ, Rogers TT. 2007. Where do you know what you know? The

876 representation of semantic knowledge in the human brain. Nature Reviews, December 2007. DOI:

$877 \quad 10.1038 / \mathrm{nrn} 2277$

878 Perelman C. 1981. Il dominio retorico: Retorica e argomentazione. Torino: Einaudi. [Or. ed.:

879 Perelman C. 1977. L'empire rhétorique : Rhétorique et argumentation. Paris: Vrin.]

880 Perfors A, Tenenbaum JB, Griffiths TL, Xu F. 2011. A tutorial introduction to Bayesian models of

881 cognitive development. Cognition Volume 120, Issue 3, September 2011: 302-321. DOI :

882 10.1016/j.cognition.2010.11.015.

883 Pettigiani MG, Sica S. 2003. La comunicazione interumana. Milano: F. Angeli.

884 Pezzulo G, Baldassarre G, Butz MV, Castelfranchi C, Hoffmann J. 2006. An analysis of the

885 Ideomotor principle and TOTE. In: Butz MV, Sigaud O, Pezzulo G, Baldassarre G, ed.

886 Anticipatory Behavior in Adaptive Learning Systems: Advances in Anticipatory Processing.

887 Berlin: Springer, 73-93. 
888 Pobric G, Jefferies E, Lambon Ralph Ma. 2010. Category-specific versus category-general

889 semantic impairment induced by Transcranial Magnetic Stimulation. Current Biology 20, $964-$

890 968, May 25, 2010. DOI: 10.1016/j.cub.2010.03.070

891 Poincaré JH. 2003 (1902). La scienza e l’ipotesi. Milano: Bompiani. [English ed.: Poincaré

892 JH.1905. Science and Hypothesis. London-Newcastle O. T.: Walter Scott Publishing.]

893 Poincaré JH.1997 (1908). Scienza e metodo. Torino: Einaudi. [English ed.: Poincaré JH. 1914.

894 Science and method. London-Edinburgh-Dublin-New York: Nelson.]

895 Pulvermüller F, Moseley RL, Egorova N, Shebani Z, Boulenger V. 2014. Motor cognition-motor

896 semantics: Action-perception theory of cognition and communication. Neuropsychologia 55

897 (2014) 71-84. DOI: 10.1016/j.neuropsychologia.2013.12.002.

898 Rizzolatti G, Craighero L. 2004. The mirror-neuron system. Annual review of Neuroscience 27:

899 169-192. DOI: 10.1146/annurev.neuro.27.070203.144230.

900 Rizzolatti G, Fabbri-Destro M. 2008. The mirror system and its role in social cognition. Current

901 Opinion in Neurobiology 18: 1-6. DOI: 10.1016/j.conb.2008.08.001.

902 Rizzolatti G, Fogassi L, Gallese V. 2001. Neurophysiological mechanisms underlying the

903 understanding and imitation of action. Nature Reviews Neuroscience 2: 661-670. DOI:

$904 \quad 10.1038 / 35090060$.

905 Rizzolatti G, Sinigaglia C. 2006. So quel che fai: Il cervello che agisce e i neuroni specchio.

906 Milano: Cortina.

907 Rizzolatti G, Vozza L. 2008. Nella mente degli altri. Milano: Zanichelli. 
908 Rose S. 2005. Il cervello del XXI Secolo: Spiegare, curare e manipolare la mente. Torino: Codice

909 Edizioni. [Or. ed.: Rose S. 2005. The 21 ${ }^{\text {st }}$ Century brain: Explaining, mending and manipulating

910 the mind. London: Jonathan Cape-Random House.]

911 Schachter S, Singer JE. 1962. Cognitive, social and physiological determinants of emotional

912 state. Psychological Review Vol.69, No.5, September 1962.

913 Sclavi M. 2003. Arte di ascoltare e mondi possibili. Milano: Bruno Mondadori.

914 Speer NK, Reynolds JR, Swallow KM, Zacks JM. 2008. Reading stories activates neural

915 representations of visual and motor experiences. Psychological Science 20/8: 989-999. DOI:

916 10.1111/j.1467-9280.2009.02397.x.

917 Sperry RW. 1952. Neurology and the mind-brain problem. American Scientist 40: 290-312.

918 Stekelenburg JJ, Vroomen J. 2012. Electrophysiological evidence for a multisensory speech-

919 specific mode of perception. Neuropsychologia 50 (2012) 1425-1431. DOI:

920 10.1016/j.neuropsychologia.2012.02.027.

921 Stöcker C, Hoffmann J. 2004. The ideomotor principle and motor sequence acquisition: Tone

922 effects facilitate movements chunking. Psychological research 68: 126-137. DOI:

$92310.1007 / \mathrm{s} 00426-003-0150-9$.

924 Tenenbaum JB, Kemp C, Griffiths TL, Goodman ND. 2011. How to Grow a Mind: Statistics, 925 Structure, and Abstraction. Science 331 : 1279. DOI: 10.1126/science.1192788. 
926 Tettamanti M, Buccino G, Saccuman MC, Gallese V, Danna M, Scifo P, Fazio F, Rizzolatti G,

927 Cappa SF, Perani D. 2005. Listening to action-related sentences activates fronto-parietal motor

928 circuits. Journal of Cognitive Neuroscience 17(2): 273-281.

929 Thibodeau PH, Boroditsky, L. 2011. Metaphors we think with: the role of metaphor in reasoning.

$930 \quad$ PLoS ONE 6(2): e16782. DOI:10.1371/journal.pone.0016782.

931 Thibodeau PH, Boroditsky, L. 2013. Natural language metaphors influence reasoning. PLoS

932 ONE 8(1): e52961. DOI:10.1371/journal.-pone.0052961.

933 Tsay CJ. 2013. Sight over sound in the judgement of music performance. Proceedings of the

934 National Academy of Sciences 2013110 (36) 14580-14585. DOI: 10.1073/pnas.1221454110.

935 Turing A.1950. Computing machinery and intelligence. Mind 59: 433-460. DOI:

936 10.1093/mind/LIX.236.433. Available: http://www.csee.umbc.edu/courses/471/papers/turing.pdf

937 (accessed 1 June 2015).

938 Verbeke M, Van Asch V, Morante R, Frasconi P, Daelemans W, De Raedt L. 2012. A statistical

939 relational learning approach to identifying evidence based medicine categories. Proceedings of

940 the 2012 Conference on Empirical Methods in Natural Language Processing and Computational

941 Natural Language Learning (EMNLP-CoNLL 2012), Jeju, Korea July 12-14, 2012. Available:

942 https://lirias.kuleuven.be/bitstream/123456789/350664/1/VerbekeEtAl_EMNLP2012.pdf

943 (accessed 1 June 2015).

944 Vitevitch MS, Sereno J, Jongman A, Goldstein R. 2013. Speaker Sex Influences Processing of

945 Grammatical Gender. PLoS ONE 8(11): e79701. DOI: 10.1371/journal.pone.0079701. 
946 von Baeyer HC. 2013. Can Quantum Bayesianism Fix the Paradoxes of Quantum Mechanics?

947 Scientific American, June 2013.

948 Watzlawick P, Beavin Bavelas J, Jackson DD. 1971. Pragmatica della comunicazione umana.

949 Roma: Astrolabio-Ubaldini. [Or. ed.: Watzlawick P, Beavin Bavelas J, Jackson DD. 1967.

950 Pragmatics of human communication. New York: Norton.]

951 Watzlawick P. 1987. If you desire to see, learn how to act. In: Zeig JK, ed. The evolution of

952 psychotherapy. New York: Brunner/Mazel, 91-100.

953 Zeilinger A. 2012. La danza dei fotoni - Da Einstein al teletrasporto quantistico. Torino, Codice.

954 [Or. ed.: Zeilinger A. 2010. Dance of the Photons - From Einstein to quantum teleportation. New

955 York: Farrar Straus \& Giroux.]

956 Zeki S. 2010. Splendori e miserie del cervello: L'amore, la creatività e la ricerca della felicità.

957 Torino: Codice Edizioni. [Or. ed.: Zeki S. 2009. Splendours and miseries of the brain: Love,

958 creativity and the quest for human happiness. Chichester: Wiley-Blackwell.]

959 Zhong CB, Bohns VK, Gino F. 2010. Good lamps are the best police: darkness increases

960 dishonesty and self-interested behaviour. Psychological Science Published online 29 January

961 2010. DOI: 10.1177/0956797609360754.

962 Zipoli Caiani S. 2013. Cognizione incorporata. APhEx - Portale italiano di filosofia analitica

963 (Periodico online - ISSN 2036-9972) 8, 2013. Available:

964 http://www.aphex.it/public/file/Content20140103 APhEx8,2013Cognizioneincorporata-

965 Zipoli.pdf (accessed 1 June 2015). 
966 Zuberbühler K. 2005. The phylogenetic roots of language: evidence from Primate communication

967 and cognition. Current directions in psychological science 2005, 14:126. DOI: 10.1111/j.0963-

968 7214.2005.00357.x. 


\section{1}

The "megaphone-shape" model.

If the interpretation of a message should be linked only to the conscious processing of its information content, then we would expect a uniform interpretation, given that the source information is absolutely identical for all the participants. On the contrary, a wide scatter is always observed and its process can be represented with a "megaphone-shape" metaphor: information would be homogeneously processed but differently interpreted.

\section{The « MEGAPHONE »}

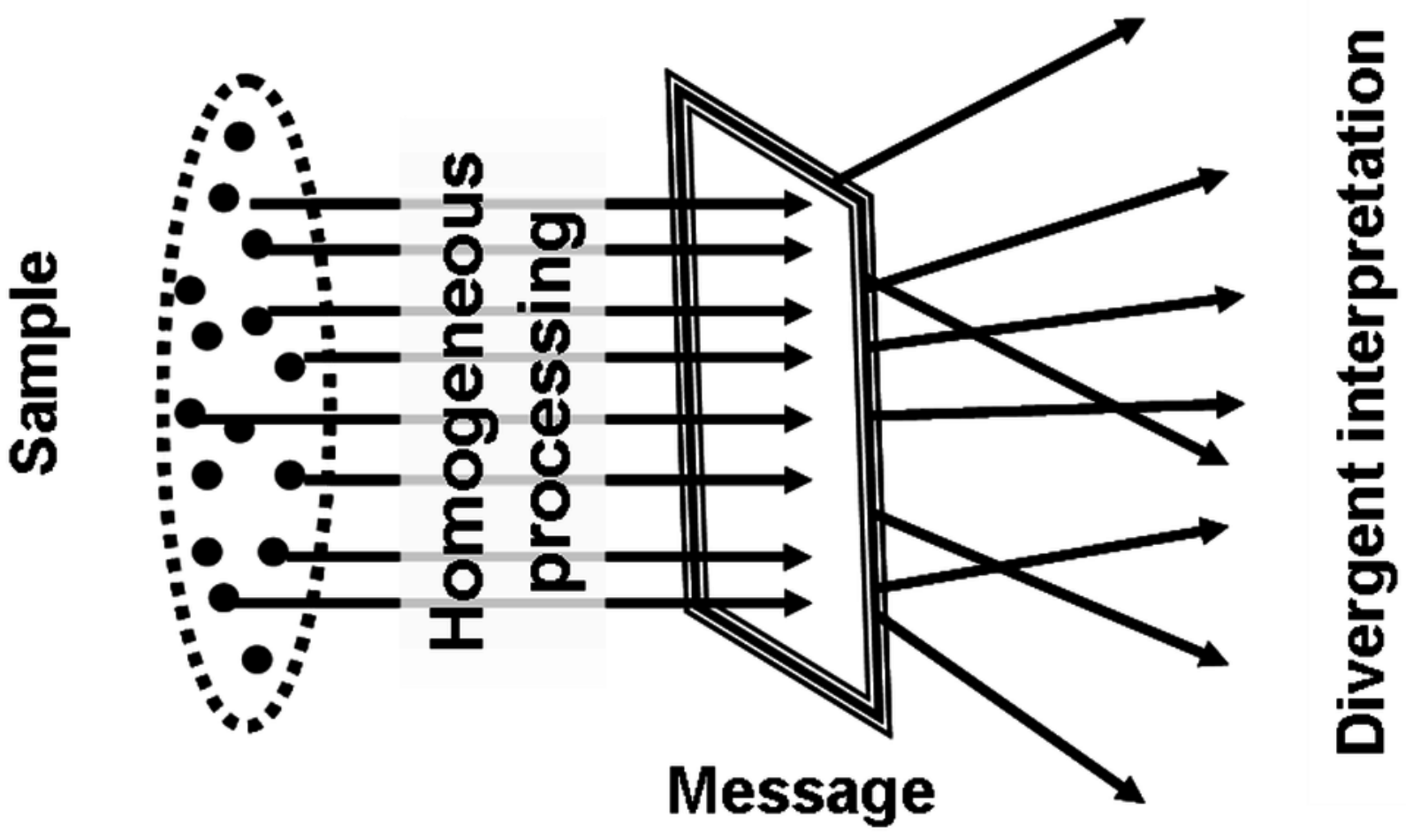


2

The "funnel-shape" model.

If the systematically observed scattering of message interpretations would be based on the scattering at "disassembling" step, we could expect that focusing on one same component would be followed by a convergent interpretation of it, as shown in this figure through the metaphor of the "funnels". This is the opposite of the "megaphone-shape" metaphor shown in Fig. 1.

\section{The « FUNNELS »}

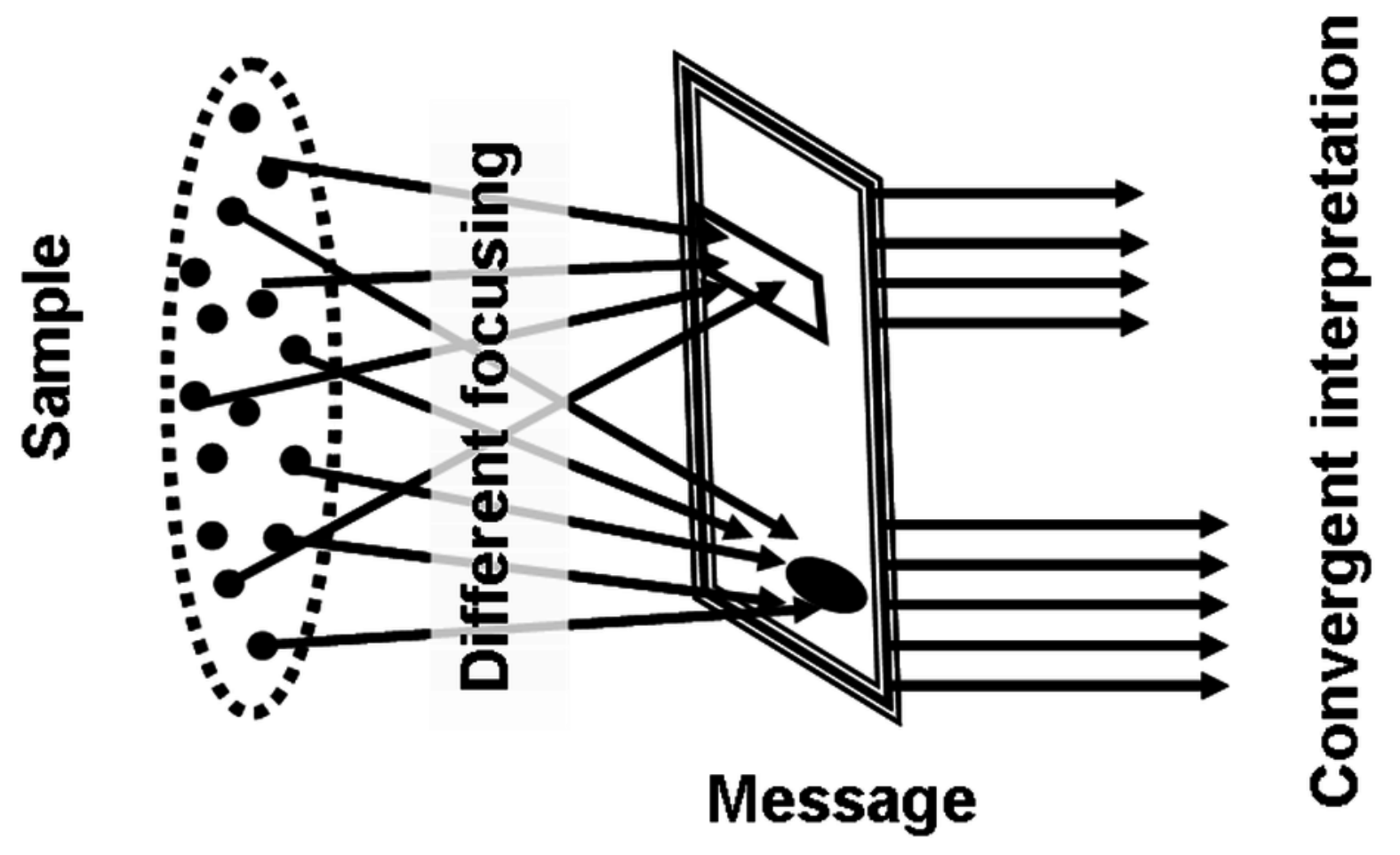




\section{3}

The "hourglass-shape" model.

This figure displays a metaphor representing the on-field observed process of message interpretation. Two kinds of scatter co-exist, manifesting themselves in sequence: the first one regards dispersion during the focusing on the components ("disassembling"); the second one regards the interpretation of the focused components (conscious information processing).

\section{The « HOURGLASSES »}

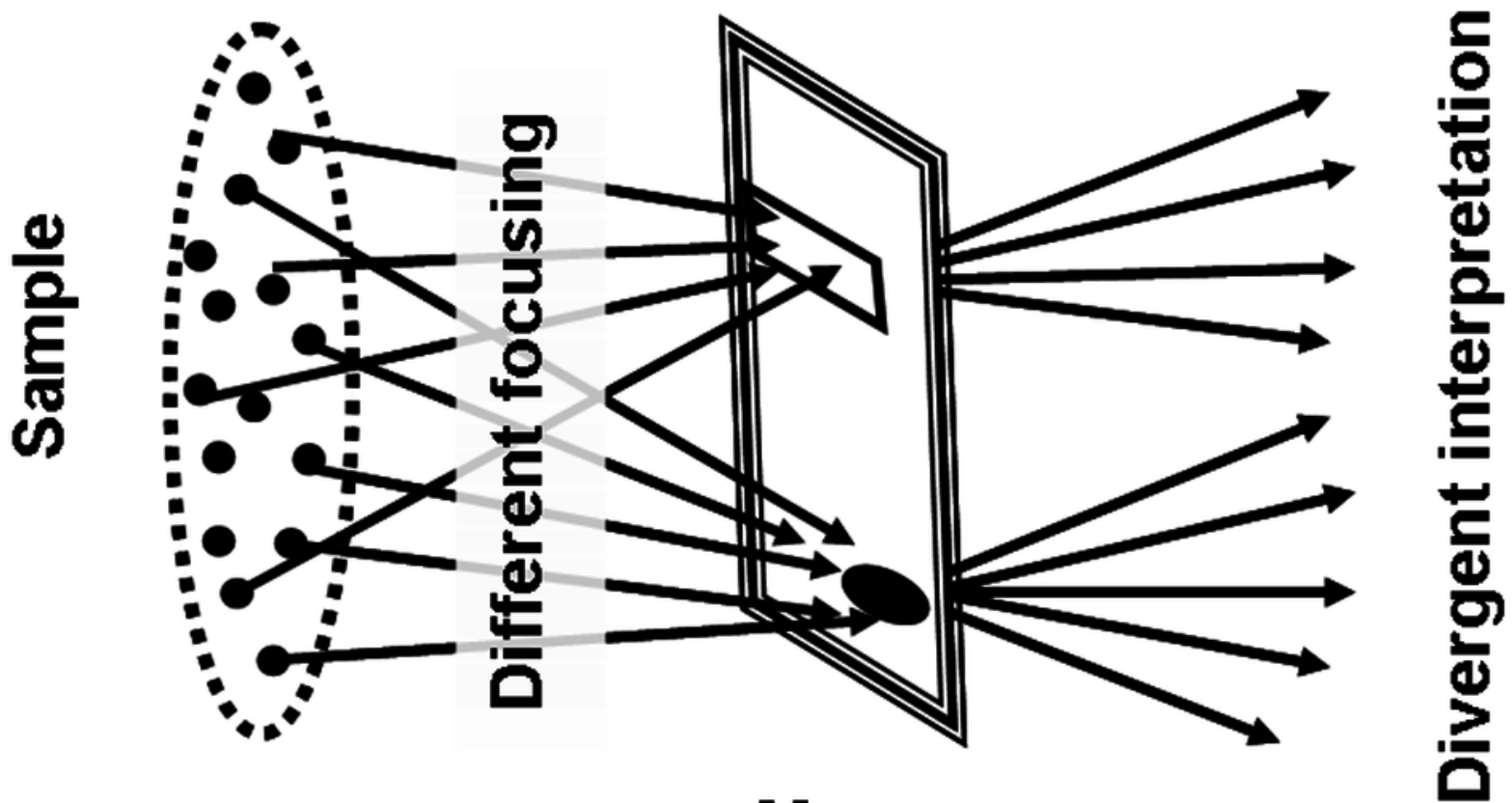

Message 
4

Scheme of the process of written message interpretation.

LEGEND: $S=$ Sender; $R=$ Receiver; $1-2-3=$ Progressive steps of the process. This figure presents our hypothesis about how a written message is understood by the receiver.

Message production (performed by the sender) is not detailed. The process of interpretation is made up by three sub-processes, in a cascade. The automatic reaction on perceptual basis (step \#2) is followed by the conscious information processing (step \#3). The step \#1 is decoding, given that the words must be, at first, recognized in order to be interpreted.

\section{The Process of Written Message Understanding}

Mess. production

Message interpretation
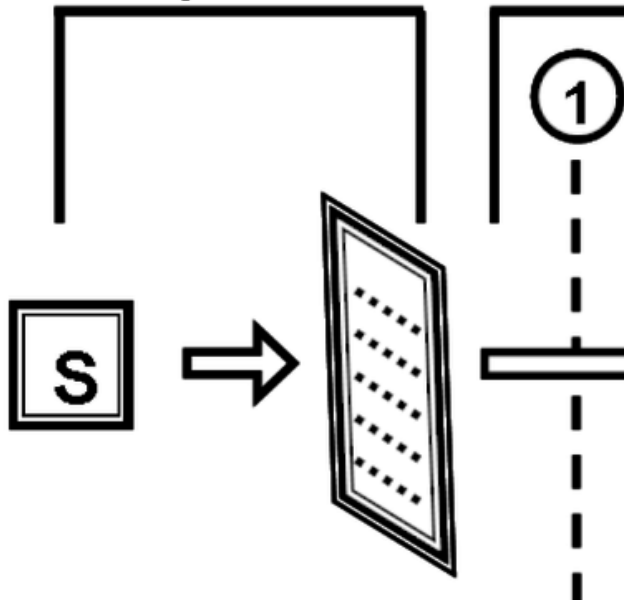

(1)
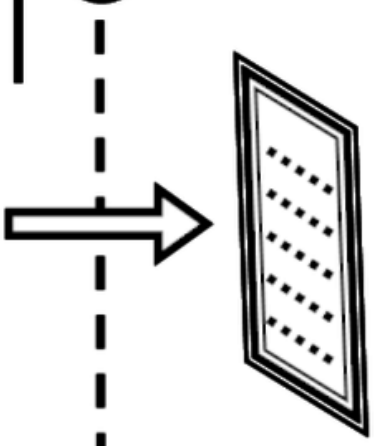

(2)
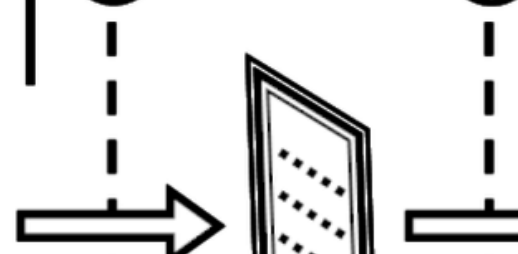

Source message

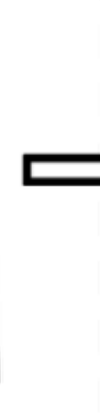

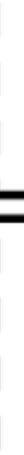
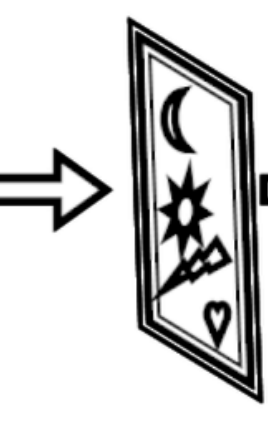

3

Decoding

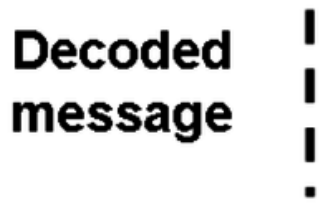

Embodied message

Perception / Automatic reaction
Conscious processing 
5

Sample distribution with respect to coherence levels / Undifferentiated Total Sample

LEGEND: $L=$ Low; $L M=$ Low-Medium; $M G=$ Medium-Great; $G=$ Great level of coherence.

This histogram shows the distribution of ALL respondents according to the coherence (expressed through the coherence indicator) between, on the one hand, their interpretations of Messages \#4/H (the "Hard" version) and \#4/S (the "Softer" version); on the other hand, their final "H-or-S" choice. Data is shown for the undifferentiated total sample. The L level results over-represented with respect to what expected.

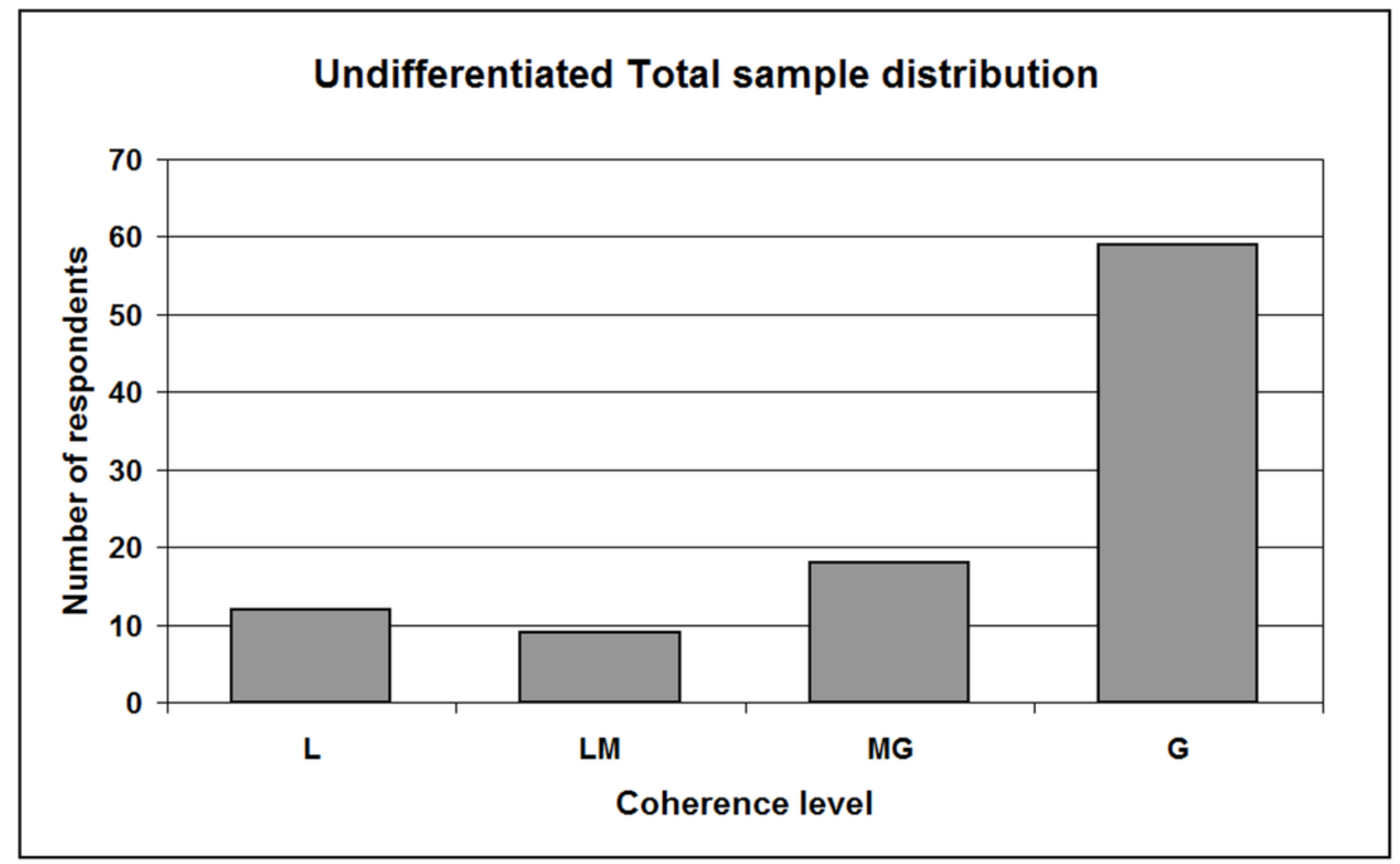


6

Sample percent distribution with respect to coherence levels / Comparing " $\mathrm{H}$ " and "S" choosers - Total Sample.

LEGEND: $L=$ Low; $L M=$ Low-Medium; $M G=$ Medium-Great; $G=$ Great level of coherence.

This histogram shows the percent distribution of ALL respondents according to the coherence (expressed through the coherence indicator) between, on the one hand, their interpretations of Messages \#4/H (the "Hard" version) and \#4/S (the "Softer" version); on the other hand, their final "H-or-S" choice. Data is shown separately for " $\mathrm{H}$ " and "S" choosers. Distributions result significantly different (Chi-squared test and Fisher's Exact test: $p=0.000$ ).

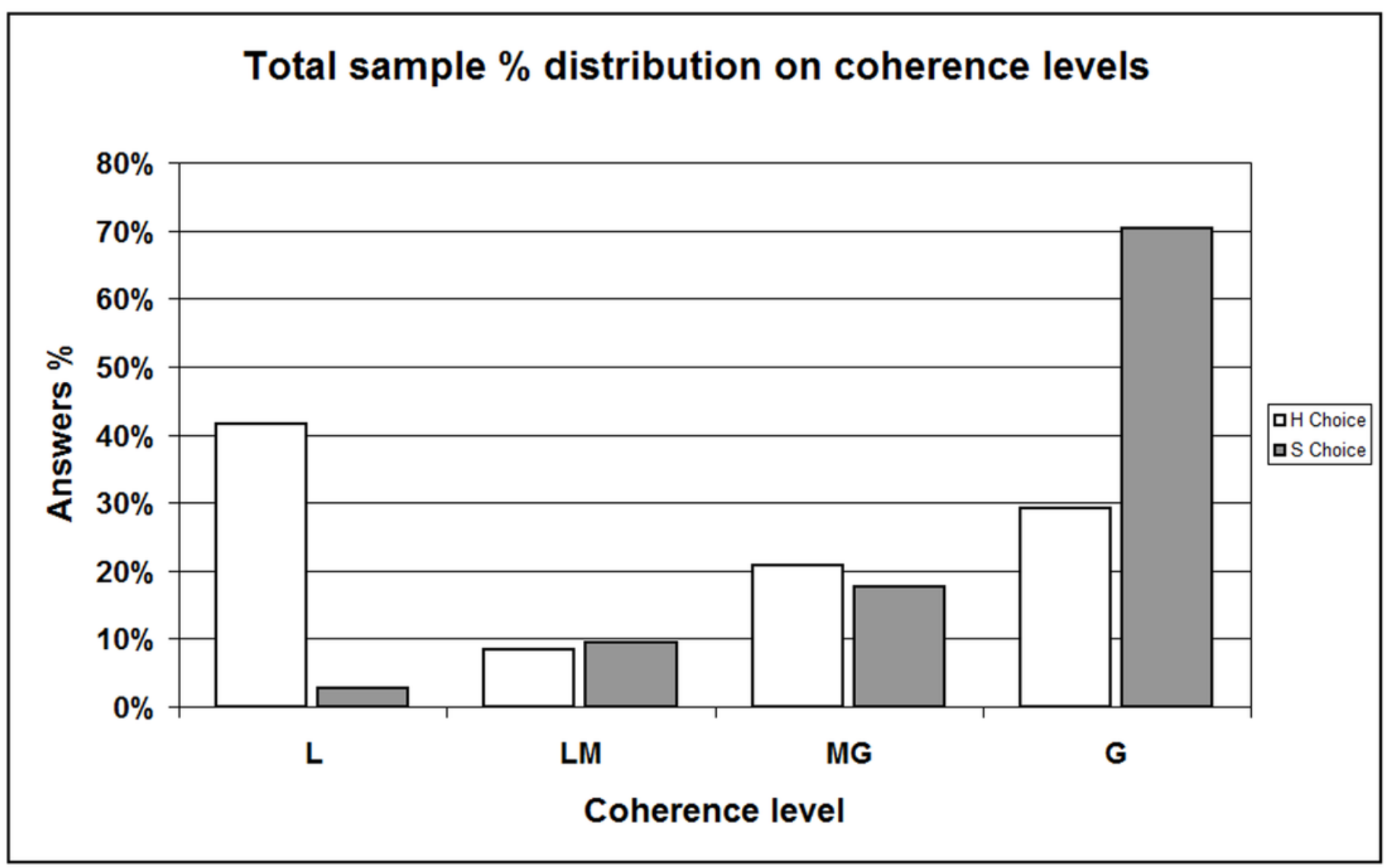


7

Sample percent distribution with respect to coherence levels / Comparing " $\mathrm{H}$ " and "S" choosers - Sub-sample "AGE".

LEGEND: $L=$ Low; $L M=$ Low-Medium; $M G=$ Medium-Great; $G=$ Great level of coherence. This histogram shows the percent distribution of respondents belonging to sub-sample "AGE" (30 years, and over, old persons) according to the coherence (expressed through the coherence indicator) between, on the one hand, their interpretations of Messages \#4/H (the "Hard" version) and \#4/S (the "Softer" version); on the other hand, their final "H-or-S" choice. Data is shown separately for " $\mathrm{H}$ " and " $\mathrm{S}$ " choosers. Distributions result significantly different (Chi-squared test and Fisher's Exact test: $p=0.000$ ).

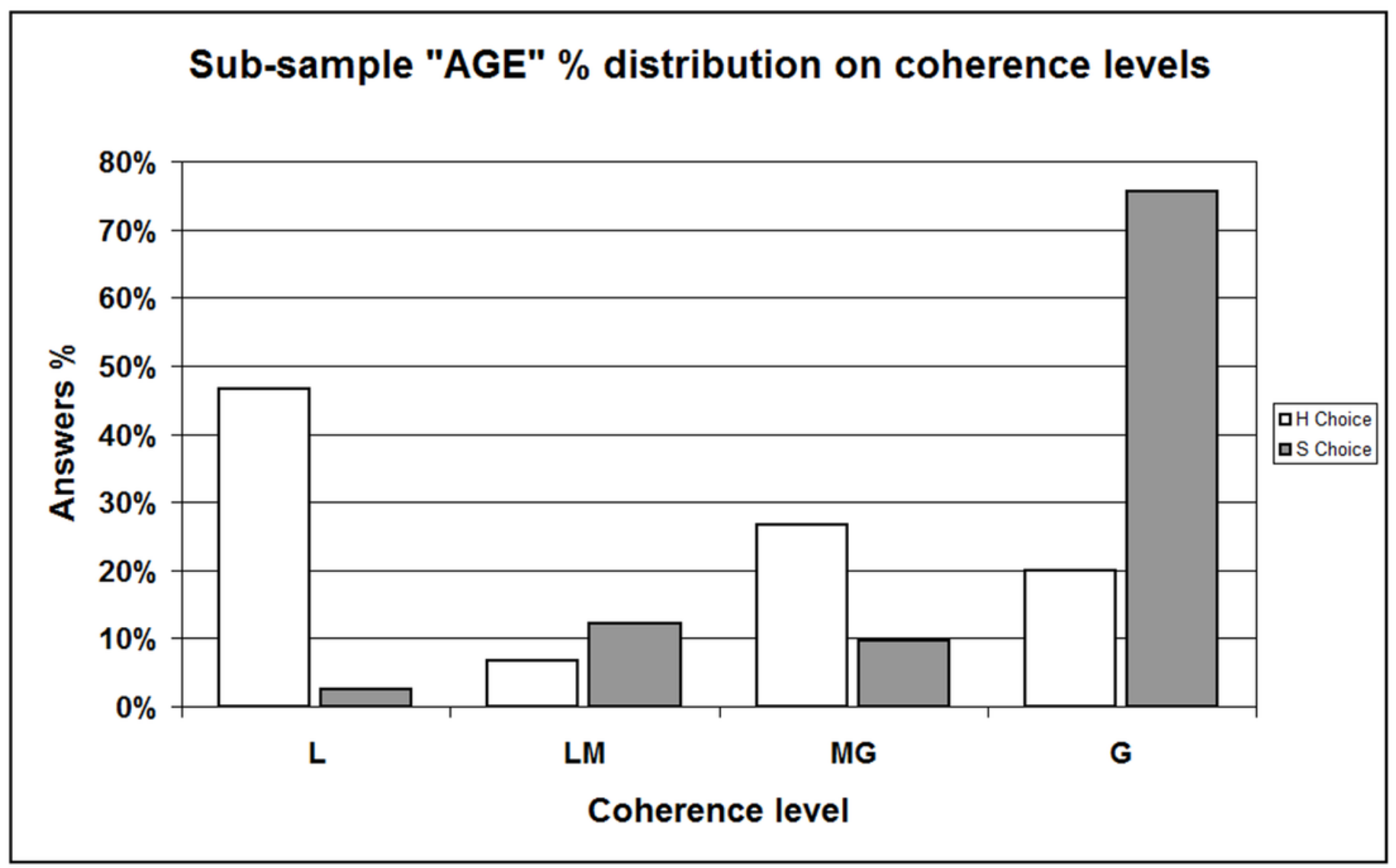


8

Sample percent distribution with respect to coherence levels / Comparing " $\mathrm{H}$ " and " $\mathrm{S}$ " choosers - Sub-sample "EMPLOYMENT".

LEGEND: $L=$ Low; $L M=$ Low-Medium; $M G=$ Medium-Great; $G=$ Great level of coherence.

This histogram shows the percent distribution of respondents belonging to sub-sample "EMPLOYMENT" (workers only, students and unemployed excluded) according to the coherence (expressed through the coherence indicator) between, on the one hand, their interpretations of Messages \#4/H (the "Hard" version) and \#4/S (the "Softer" version); on the other hand, their final "H-or-S" choice. Data is shown separately for " $\mathrm{H}$ " and "S" choosers. Distributions result significantly different (Chi-squared test and Fisher's Exact test: $p=0.000)$.

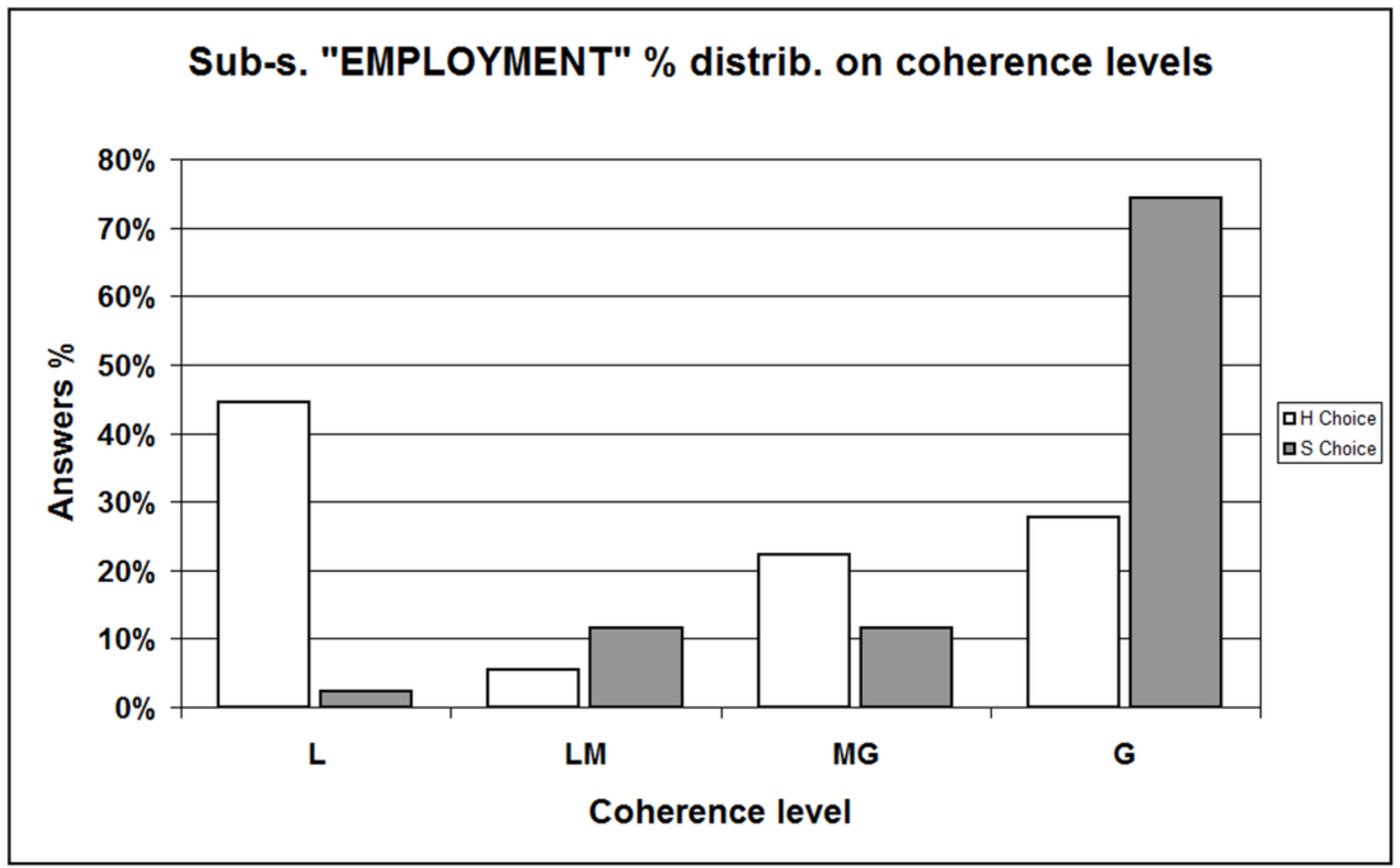




\section{Table $\mathbf{1}$ (on next page)}

Main features of the sample (total sample).

The table provides a quantitative description of the total sample with regards to age (left columns), education level (central columns) and employment (right columns) of the participants; see Legends for the used symbols. Data is shown either as values or in percentage and split down by gender ( $M=$ males; $F=$ Females $)$. 


\begin{tabular}{|c|c|c|c|c|c|c|c|c|c|c|c|c|c|c|c|c|c|}
\hline \multicolumn{6}{|l|}{ Age } & \multicolumn{6}{|c|}{ Education } & \multicolumn{6}{|c|}{ "Employment } \\
\hline \multirow[t]{2}{*}{ Bin } & \multicolumn{2}{|l|}{$M$} & \multicolumn{2}{|l|}{$F$} & \multirow[t]{2}{*}{ Tot } & \multirow[t]{2}{*}{ Bin } & \multicolumn{2}{|l|}{$M$} & \multicolumn{2}{|l|}{$F$} & \multirow[t]{2}{*}{ Tot } & \multirow[t]{2}{*}{ Bin } & \multicolumn{2}{|l|}{$M$} & \multicolumn{2}{|l|}{$F$} & \multirow[t]{2}{*}{ Tot } \\
\hline & Val. & $\%$ & Val. & $\%$ & & & Val. & $\%$ & Val. & $\%$ & & & Val. & $\%$ & Val. & $\%$ & \\
\hline $\bar{A}$ & 10 & 23.8 & 32 & 76.2 & 42 & $\mathrm{El}$ & 1 & 25.0 & 3 & 75.0 & 4 & A & 16 & 47.1 & 18 & 52.9 & 34 \\
\hline $\mathrm{B}$ & 11 & 36.7 & 19 & 63.3 & 30 & $\mathrm{Dg}$ & 18 & 46.2 & 21 & 53.8 & 39 & $\mathrm{~B}$ & 6 & 85.7 & 1 & 14.3 & 7 \\
\hline $\mathrm{C}$ & 7 & 46.7 & 8 & 53.3 & 15 & $\mathrm{Gr}$ & 18 & 30.5 & 41 & 69.5 & 59 & $\mathrm{C}$ & 6 & 31.6 & 13 & 68.4 & 19 \\
\hline $\mathrm{D}$ & 9 & 60.0 & 6 & 40.0 & 15 & -- & -- & -- & -- & -- & -- & $\mathrm{D}$ & 1 & 20.0 & 4 & 80.0 & 5 \\
\hline-- & -- & -- & -- & -- & -- & -- & -- & -- & -- & -- & -- & $\mathrm{E}$ & 5 & 17.2 & 24 & 82.8 & 29 \\
\hline -- & -- & -- & -- & -- & -- & - & - & -- & -- & -- & -- & $\mathrm{F}$ & 3 & 37.5 & 5 & 62.5 & 8 \\
\hline Tot & 37 & & 65 & & 102 & Tot & 37 & & 65 & & 102 & Tot & 37 & & 65 & & 102 \\
\hline
\end{tabular}

Legend (age): $\mathrm{A}=18-29$ yу ; $\mathrm{B}=30-39$ yу ; $\mathrm{C}=40-49$ yу $; \mathrm{D}=50$ yy and over

Legend (education): $\mathrm{El}=$ Elementary level; $\mathrm{Dg}=$ High School degree $; \mathrm{Gr}=$ Graduates $/$ Post-graduates

Legend (employment): $\mathrm{A}=$ Line workers ; $\mathrm{B}=$ Managers ; $\mathrm{C}=$ Graduated technicians/Professionals ; $\mathrm{D}=\mathrm{Artisans} /$ Entrepreneurs ; $\mathrm{E}=\mathrm{Students} \mathrm{;} \mathrm{F}=\mathrm{Unemployed} / \mathrm{Others}$ 


\section{Table 2 (on next page)}

Main features of the sample (sub-sample "Age", >29yy).

The table provides a quantitative description of the sub-sample "Age" (only participants 30 years, and over, old) with regards to age (left columns), education level (central columns) and employment (right columns) of the participants; see Legends for the used symbols. Data is shown either as values or in percentage and split down by gender $(\mathrm{M}=$ males; $\mathrm{F}=$ Females). 


\begin{tabular}{|c|c|c|c|c|c|c|c|c|c|c|c|c|c|c|c|c|c|}
\hline \multicolumn{6}{|c|}{ Age } & \multicolumn{6}{|c|}{ Education } & \multicolumn{6}{|c|}{ Employment } \\
\hline \multirow[t]{2}{*}{ Bin } & \multicolumn{2}{|l|}{$M$} & \multicolumn{2}{|l|}{$F$} & \multirow[t]{2}{*}{ Tot } & \multirow[t]{2}{*}{ Bin } & \multicolumn{2}{|l|}{$M$} & \multicolumn{2}{|l|}{$F$} & \multirow[t]{2}{*}{ Tot } & \multirow[t]{2}{*}{ Bin } & \multicolumn{2}{|l|}{$M$} & \multicolumn{2}{|l|}{$F$} & \multirow[t]{2}{*}{ Tot } \\
\hline & Val. & $\%$ & Val. & $\%$ & & & Val. & $\%$ & Val. & $\%$ & & & Val. & $\%$ & Val. & $\%$ & \\
\hline A & l & l & l & / & / & El & 1 & 25.0 & 3 & 75.0 & 4 & A & 14 & 46.7 & 16 & 53.3 & 30 \\
\hline B & 11 & 36.7 & 19 & 63.3 & 30 & $\mathrm{Dg}$ & 12 & 52.2 & 11 & 47.8 & 23 & $\mathrm{~B}$ & 6 & 85.7 & 1 & 14.3 & 7 \\
\hline $\mathrm{C}$ & 7 & 46.7 & 8 & 53.3 & 15 & $\mathrm{Gr}$ & 14 & 42.4 & 19 & 57.6 & 33 & $\mathrm{C}$ & 6 & 37.5 & 10 & 62.5 & 16 \\
\hline $\mathrm{D}$ & 9 & 60.0 & 6 & 40.0 & 15 & -- & -- & -- & -- & -- & -- & $\mathrm{D}$ & 1 & 25.0 & 3 & 75.0 & 4 \\
\hline-- & -- & -- & -- & -- & -- & -- & -- & -- & -- & -- & -- & $\mathrm{E}$ & $\mathbf{0}$ & 0.0 & 2 & 100 & 2 \\
\hline-- & -- & -- & -- & -- & -- & -- & -- & -- & -- & -- & -- & $\mathrm{F}$ & 0 & 0.0 & 1 & 100 & 1 \\
\hline Tot & 27 & & 33 & & 60 & Tot & 27 & & 33 & & 60 & Tot & 27 & & 33 & & 60 \\
\hline
\end{tabular}

Legend (age): $\mathrm{A}=18-29$ yу ; $\mathrm{B}=30-39$ yу ; $\mathrm{C}=40-49$ yу ; $\mathrm{D}=50$ yy and over

Legend (education): El = Elementary level; Dg = High School degree ; Gr = Graduates / Post-graduates

Legend (employment): A = Line workers ; B = Managers ; $\mathrm{C}=$ Graduated technicians/Professionals ; D = Artisans/Entrepreneurs ; E = Students ; $\mathrm{F}=\mathrm{Unemployed} /$ Others 


\section{Table 3 (on next page)}

Main features of the sample (sub-sample "Employment", job owners).

The table provides a quantitative description of the sub-sample "Employment" (participants with a regular employment only) with regards to age (left columns), education level (central columns) and employment (right columns) of the participants; see Legends for the used symbols. Data is shown either as values or in percentage and split down by gender ( $M=$ males; $\mathrm{F}=$ Females). 


\begin{tabular}{|c|c|c|c|c|c|c|c|c|c|c|c|c|c|c|c|c|c|}
\hline \multicolumn{6}{|l|}{ Age } & \multicolumn{6}{|c|}{ Education } & \multicolumn{6}{|c|}{ Employment } \\
\hline \multirow[t]{2}{*}{ Bin } & \multicolumn{2}{|l|}{$M$} & \multicolumn{2}{|l|}{$F$} & \multirow[t]{2}{*}{ Tot } & \multirow[t]{2}{*}{ Bin } & \multicolumn{2}{|l|}{$M$} & \multicolumn{2}{|l|}{$F$} & \multirow[t]{2}{*}{ Tot } & \multirow[t]{2}{*}{ Bin } & \multicolumn{2}{|l|}{$M$} & \multicolumn{2}{|l|}{$F$} & \multirow[t]{2}{*}{$T o$} \\
\hline & Val. & $\%$ & Val. & $\%$ & & & Val. & $\%$ & Val. & $\%$ & & & Val. & $\%$ & Val. & $\%$ & \\
\hline $\mathrm{A}$ & 2 & 25.0 & 6 & 75.0 & 8 & $\mathrm{El}$ & 1 & 25.0 & 3 & 75.0 & 4 & A & 16 & 47.1 & 18 & 52.9 & 34 \\
\hline B & 11 & 40.7 & 16 & 59.3 & 27 & Dg & 13 & 52.0 & 12 & 48.0 & 25 & B & 6 & 85.7 & 1 & 14.3 & 7 \\
\hline $\mathrm{C}$ & 7 & 46.7 & 8 & 53.3 & 15 & Gr & 15 & 41.7 & 21 & 58.3 & 36 & $\mathrm{C}$ & 6 & 31.6 & 13 & 68.4 & 19 \\
\hline $\mathrm{D}$ & 9 & 60.0 & 6 & 40.0 & 15 & -- & -- & -- & -- & -- & -- & $\mathrm{D}$ & 1 & 20.0 & 4 & 80.0 & 5 \\
\hline -- & -- & -- & -- & -- & -- & -- & -- & -- & -- & -- & -- & $\mathrm{E}$ & 1 & 1 & 1 & 1 & 1 \\
\hline-- & -- & -- & -- & -- & -- & -- & -- & -- & -- & -- & -- & $\mathrm{F}$ & 1 & 1 & I & I & 1 \\
\hline Tot & 29 & & 36 & & 65 & Tot & 29 & & 36 & & 65 & Tot & 29 & & 36 & & 65 \\
\hline
\end{tabular}

Legend (age): $\mathrm{A}=18-29$ yy ; $\mathrm{B}=30-39$ yy ; $\mathrm{C}=40-49$ yy ; $\mathrm{D}=50$ yy and over

Legend (education): $\mathrm{El}=$ Elementary level; $\mathrm{Dg}=$ High School degree ; $\mathrm{Gr}=$ Graduates $/$ Post-graduates

Legend (employment): A = Line workers ; B = Managers ; C = Graduated technicians/Professionals ; D = Artisans/Entrepreneurs ; E = Students ; F = Unemployed/Others 


\section{Table 4 (on next page)}

Full-text of Message \#4 two versions (H/"Hard and S/"Softer") and Message \#5.

This table presents the full-text two versions of Message \#4, labelled as "Hard" (the original version by YY) and "Softer" (the version suggested by one colleague of his). The full-text final Message \#5 is added. 


\section{Message \#4/H (the "Hard" version)}

From: YY (Project Account for the heating plant works)

To: XX (Employee in one of the offices affected by the works)

$\mathrm{Cc}$ : ZZ (Office referent for the works)

Sent: ... [date] [hour]

Subject: R: heating plant

\section{Dear Mrs. XX,}

I want to premise that, for the sake of a wise management of the work process, intended to optimize the utilization of our Corporation resources (exactly, in order to avoid wasting public money):

- Before Project start, I asked the Director of your structure (B wing of the building), Dr. $\mathrm{KK}$, to put a specific person in charge of controlling the work's progress;

- As far as I am concerned, the indicated person is, and will remain, Dr. ZZ;

- Dr. ZZ carefully planned the project development steps with us;

- Each office, situated in the B wing of the building, has been already supplied with heating systems (hardware), fully complying with the timetable agreed with Mrs. ZZ;

- The heating plant is now working, even though in provisional mode.

I do recommend you to send any communication, concerning the mentioned Project, to the specific person in charge of controlling, in order to avoid (as already happened) message exchange with personnel that is not directly and formally involved within the process.

However, I inform you that, at the moment, the works under discussion have been suspended, in order to enable the provisioning of the plant-control software. It will manage automatically the heating system in the offices, including yours, regulating the warm air diffusion (in order, as said above, to reduce any waste of money).

As soon as the software will be installed by the contractor, the works will come to end. By the way, in this phase they should not affect the rooms situated in the B wing of the building at all, but only the thermo station.

All quantitative and qualitative controls, requested by the CHK form [formal inspection document], will be carried out after the end of the works and just before their compliance to fixed quality standards will be attested, as prescribed by the current rules.

This said, I have found your objections very interesting. For this reason, once the real existence of the problems you have marked will be assessed, I will certainly solve them as a part of my duty.

Yours sincerely

The Project Account / Arch. YY - [Corporation branch] .................
Message \#4/S (the "Softer" version) - Message \#5

\section{$\underline{\operatorname{Msg} \text { \#4/S }}$}

From: YY (Project Account for heating plant works)

To: XX (Employee in one of the offices affected by the works)

Cc: ZZ (Office referent for the works)

Sent: ... [date] [hour $]$

Subject: R: heating plant

\section{Dear Mrs. XX,}

I remember your last message, which I have already answered, and now I really thank you for this new one. In fact, we do believe that the attention of our colleagues, on field operating with structures and plants we provide, is fundamental to complete our tasks at best.

In order to optimize our contribution, I have been since the beginning asking for a unique person in charge of controlling the works, accounted for your office's building. This person is Doctor ZZ (I might have already mentioned her in my previous answer even though, at present time, I am not certain about this). Her duty is to collect all the observations expressed by the staff about the work in progress, then to send it directly to my office. I think you already know her and she is going to receive a copy of the present message. I thought this would make communication easier.

Concerning your request, you can be certain that, so far, our Project has been developed by following all the technical and formal standards prescribed by the current rules. In addition, I inform you that the works are not yet concluded and final checks (along with possible inspections) are about to be carefully planned. Please, inform your colleagues about the existence of a person in charge of control and do not hesitate to contact her in the case of further observations or possible problems. As I said, she will return your indications to us; this way, I assure you they will not be ignored.

Best regards

The Project Account

Arch. YY - [Corporation branch]

\section{$\underline{\text { Msg \#5 }}$}

Thank you very much for your interest and for the information. That was very kind of you and your answer was exhaustive.

Best regards

$\mathrm{XX}$ 


\section{Table 5 (on next page)}

An example of interpretation scatter from our research.

Sixty-one individuals ( $60 \%$ of the sample), after having compared XX's Messages \#1 and \#3, answered "YES" to Question \#2 and provided 83 specifications for the changes they had detected in XX's position toward YY. The table classifies the specifications into 4 main categories and provides some examples for each one of them. 


\begin{tabular}{|c|c|c|}
\hline Category & Sub-category & Examples of participants' interpretations \\
\hline \multirow{3}{*}{$\begin{array}{l}\text { Behaviours } \\
{[\underline{7 \text { answers }}}\end{array}$} & \multirow[t]{3}{*}{ 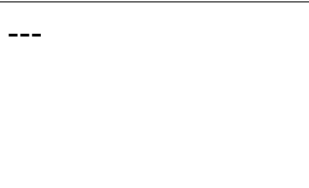 } & $\mathrm{XX}$ requests for an intervention \\
\hline & & She reports flaws \\
\hline & & She is just sending a duty communication \\
\hline \multirow{2}{*}{$\begin{array}{l}\text { Emotions } \\
{[16 \text { answers }]}\end{array}$} & \multirow[t]{2}{*}{$\mathrm{XX}$ is: } & Angry, Disturbed, Worried, Aggressive, Discouraged \\
\hline & & Brave, Impatient, Afraid \\
\hline \multirow{5}{*}{$\begin{array}{l}\text { Relations } \boldsymbol{X X}-\boldsymbol{Y} \boldsymbol{Y} \\
\text { [41 answers] }\end{array}$} & XX expresses: & Assertiveness, Aggressiveness, Superiority, Subordination \\
\hline & XX takes a position: & Tough, Technical, Neutral \\
\hline & \multirow[t]{3}{*}{$\mathrm{XX}:$} & Demands a solution \\
\hline & & Recalls YY to his duty \\
\hline & & Thwarts YY's plans \\
\hline \multirow{2}{*}{$\begin{array}{l}\text { Message form } \\
{[\underline{19 \text { answers }]}}\end{array}$} & \multirow[t]{2}{*}{ Msg \#3 is more: } & Concrete, Correct, Detailed \\
\hline & & Direct, Effective \\
\hline
\end{tabular}




\section{Table 6(on next page)}

Macro-categories of the "concrete elements" respondents have indicated as the basis of their interpretations.

The analysis of the answers to the second input of the submitted questions (respondents requested to indicate the "concrete elements" on which the interpretations they provided were based) returned the results displayed in this table. Following the accounts of the participants, their interpretations were based on aspects largely independent of the message information content. 


\begin{tabular}{|c|c|c|}
\hline Category & Sub-categories & Description \\
\hline $\begin{array}{l}\text { Information } \\
\text { content }\end{array}$ & $\begin{array}{l}\text { Information } \\
\text { content }\end{array}$ & $\begin{array}{l}\text { Summaries of the message texts and syntheses of their } \\
\text { information content, presented through respondent's own words. }\end{array}$ \\
\hline $\begin{array}{l}\text { Meaningful } \\
\text { elements }\end{array}$ & $\begin{array}{l}\text { * Words } \\
\text { * Phrases/periods }\end{array}$ & $\begin{array}{l}\text { Quotations between double quotes, referred to selected words, } \\
\text { full phrases (or parts of them) or periods. Such kind of indications } \\
\text { have been provided also through pointing the beginning and the } \\
\text { ending word of the quoted strings ("from... to..."). The string } \\
\text { length could cover up to a whole paragraph of the message (from } \\
\text { a keyboard "Enter" to the following). }\end{array}$ \\
\hline $\begin{array}{l}\text { Incidental } \\
\text { passages }\end{array}$ & $\begin{array}{l}\text { Incidental } \\
\text { passages }\end{array}$ & $\begin{array}{l}\text { Incidental strings, meaningless per se. Such strings were } \\
\text { extracted from original full phrases and quoted isolated from the } \\
\text { rest. }\end{array}$ \\
\hline $\begin{array}{l}\text { Accessory } \\
\text { elements }\end{array}$ & $\begin{array}{l}* \text { Symbols } \\
* \text { Titles/salutes } \\
\text { * Grammar } \\
\text { notations }\end{array}$ & $\begin{array}{l}\text { Complement/accessory parts of the text: punctuation marks }{ }^{*} \text {, } \\
\text { personal or professional titles used in the opening, the salutes used } \\
\text { in the closing etc.. } \\
{ }^{(*)} \text { In one of the two pilot-sessions of the survey, one message contained an } \\
\text { exclamation mark; it was specifically identified, and noted as a meaningful } \\
\text { component per se, by one of the participants. For this reason, it was } \\
\text { removed in order to limit influencing respondents. In fact, other } \\
\text { respondents successively picked up, from questionnaires now bereft of that } \\
\text { exclamation mark, quotation marks (used in certain passages of the } \\
\text { submitted messages) as a meaningful component per se. }\end{array}$ \\
\hline $\begin{array}{l}\text { Other } \\
\text { elements }\end{array}$ & Other elements & $\begin{array}{l}\text { Items unrelated to the text semantics or to the message content; a } \\
\text { tight selection is presented in Table } 7 \text {. The list is indefinite, given } \\
\text { that each item generally appears at low frequency while the range } \\
\text { of possible items is extremely widespread. Items of this kind are } \\
\text { actually unpredictable; even the lack of some content can be } \\
\text { focused and reported as a source of meaning (Table } 7 \text {, final row). }\end{array}$ \\
\hline $\begin{array}{l}\text { Whole } \\
\text { message }\end{array}$ & Whole message & $\begin{array}{l}\text { References to some overall effects produced by the message on the } \\
\text { participant (see SI Section 8.a, final part, for details). In fact, in this } \\
\text { kind of answers, participants state they cannot indicate any } \\
\text { "concrete element"; the meaning they have attributed derives from } \\
\text { a "general impression" received from the message, from the } \\
\text { message's "general tone". }\end{array}$ \\
\hline
\end{tabular}




\section{Table 7 (on next page)}

A selection of "other elements" that readers may focus on inside the messages.

The table displays a tight selection of the "other elements" (see Table 6, fifth row) focused on by respondents inside the messages. These elements are independent of the information content and, in most cases, of the message text. They are extremely various, indeed unpredictable, and return the impression that the receivers' preferences could be totally rule less. 


\begin{tabular}{|l|l||}
\hline Elements & Examples \\
\hline The POSITION of a statement & $\begin{array}{l}\text { XX explains her absence at the beginning of Msg \#3 to } \\
\text { forestall possible criticism. } \\
Y Y \text { scoffs at XX, expressing a little courtesy just at the end } \\
\text { of Msg \#4/H. }\end{array}$ \\
\hline The LENGTH of a text & $\begin{array}{l}\text { Msg \#4/H being long / Msg \#5 being short have an } \\
\text { underlying meaning. }\end{array}$ \\
\hline Dotted lists & The use of it in Msg \#4/H has a meaning. \\
\hline Type of lexicon & $\begin{array}{l}\text { The use of technical words / expressions implies precision, } \\
\text { but also suggests the intention to keep one's distance. } \\
\text { Thanking and reassuring expressions have détente effects. }\end{array}$ \\
\hline $\begin{array}{l}\text { The relational or social roles of } \\
\text { characters }\end{array}$ & $\begin{array}{l}\text { Some interpreted Msg \#4/H (the "Hard" version) as an } \\
\text { attack to XX being a woman. }\end{array}$ \\
\hline The professional roles of characters & XX not being an Account, she would not cheat. \\
\hline Grammatical observations & The verbs tense is noted as having an underlying meaning. \\
\hline LACK of content & $\begin{array}{l}Y Y \text { does NOT wonder why XX requests a control. } \\
Y Y \text { announces a solution NOT clarifying what it will be. }\end{array}$ \\
\hline \hline
\end{tabular}




\section{Table 8 (on next page)}

Statistics on indicated components.

The table displays a descriptive statistical analysis of what the respondents focus on inside the messages. The information content is expressly focused by $12.1 \%$ of respondents only ("Cont." column, "\%" row). Even if we suppose that reference to complete phrases/periods could actually mean reference to their content, the sum of "Cont." and "Phras." column \% totals would amount just to $33 \%$ of respondents, again a clear minority. 


\begin{tabular}{|c|c|c|c|c|c|c|c|c|c|c|c|}
\hline Quest. & Sym. & Titl. & Words & Incid. & Phras. & Whole & Cont. & Other & Gram. & TOT & $\%$ \\
\hline $1-\mathrm{a}$ & 1 & 7 & 46 & 55 & \begin{tabular}{|c|}
53 \\
\end{tabular} & 16 & 29 & 14 & 4 & 225 & $17.1 \%$ \\
\hline 1-b & 1 & 7 & 26 & 53 & 27 & 18 & 20 & 12 & 3 & 167 & $12.7 \%$ \\
\hline 1-c & $\mathbf{0}$ & 6 & 22 & 58 & 34 & 13 & 11 & 12 & 2 & 158 & $12.0 \%$ \\
\hline 2 & 4 & 5 & 22 & 52 & 32 & 17 & 34 & 7 & 2 & 175 & $13.3 \%$ \\
\hline $3-4 / \mathrm{H}$ & 0 & 1 & 13 & 49 & 54 & 35 & 31 & 24 & 2 & 209 & $15.9 \%$ \\
\hline $3-4 / \mathrm{S}$ & 0 & 22 & 14 & 52 & 48 & 45 & 29 & 5 & 1 & 216 & $16.4 \%$ \\
\hline Final & 2 & 14 & 17 & 30 & 28 & 50 & 6 & 22 & 0 & 169 & $12.8 \%$ \\
\hline TOT & 8 & 62 & 160 & 349 & 276 & 194 & 160 & 96 & 14 & 1,319 & $100 \%$ \\
\hline$\%$ & $0.6 \%$ & $4.7 \%$ & $12.1 \%$ & $26.4 \%$ & $20.9 \%$ & $14.7 \%$ & $12.1 \%$ & $7.3 \%$ & $1.1 \%$ & $100 \%$ & \\
\hline
\end{tabular}

LEGEND: $\underline{\text { Sym. }}=$ Symbols (punctuation marks); $\underline{\text { Titl. }}=$ Titles - Salutes (starting/closing expressions) $\underline{\text { Words }}=$ Meaningful isolated words/expressions ; $\underline{\text { Incid. }}=$ Incidental passages, meaningless per se ; $\underline{\text { Phras. }}=$ Complete phrases/periods ; $\underline{\text { Whole }}=$ General tone of the message Cont. $=$ Information content of the message ; $\underline{\text { Other }}=$ Other components of the message ; $\underline{\text { Gram. }}=$ Grammar notations, like verbs tense and similar ; $\underline{\text { TOT }}=$ Totals; $\underline{\%}=$ Percentage on totals . 


\section{Table 9 (on next page)}

Sample distribution with regards to components referred to information content.

While answering to the second input of the questions (requesting to indicate the "concrete elements" on which the interpretation was based), just the exact half of the sample indicated, at least once, information content components. In this table, the sample is distributed in bins defined through the percentage that the components referred to information content represent on the personal total of provided indications. Just for 7 people out of 102 the indications pointing at information content balance the others or prevail $(50 \%$ or more); just 1 people among them indicates information content components only. 


\begin{tabular}{|l|l|l|}
\hline Bins (\% on personal total) & N. of respondents & $\%$ \\
\hline $\mathbf{0 \%}$ & 51 & $50,0 \%$ \\
\hline $\mathbf{1 \%}-\mathbf{2 4} \%$ & 31 & $30,4 \%$ \\
\hline $\mathbf{2 5 \%}-\mathbf{4 9} \%$ & 13 & $12,7 \%$ \\
\hline $\mathbf{5 0} \%-\mathbf{9 9} \%$ & 6 & $5,9 \%$ \\
\hline $\mathbf{1 0 0 \%}$ & 1 & $1,0 \%$ \\
\hline TOTAL & 102 & $100,0 \%$ \\
\hline
\end{tabular}




\section{Table $\mathbf{1 0}$ (on next page)}

Interpretation scatter referred to one component (the incidental passage of Message \#1).

The table displays the result of classifying the interpretations given by a subset of 53 individuals ( $52 \%$ of the sample) to one component of Message \#1. These respondents, even though focusing on that same component (the incidental passage “...we would be pleased if at least once..."), have nonetheless dispersed their interpretations. This means that not even the "funnel-shape" model (Fig. 2) could result acceptable. 


\begin{tabular}{|c|c|}
\hline Category & Examples of participants' interpretations \\
\hline \multirow{3}{*}{$\begin{array}{l}\text { “... we'd be pleased...” } \\
{[\underline{32 \text { quotations }]}}\end{array}$} & Aggressiveness; Office duty expression; Informality; Irony \\
\hline & Just a request; Sarcasm; Highlighting XX's subordinate role \\
\hline & Expression of alternative visions \\
\hline \multirow{3}{*}{$\begin{array}{l}\text { “... if at least once...” } \\
{[17 \text { quotations }]}\end{array}$} & Conflict; Doubt on YY's reliability; Expression of courtesy \\
\hline & Taunting; Request for attention; Request for information \\
\hline & A reminder; Stimulus to organization top management \\
\hline \multirow{6}{*}{$\begin{array}{l}\text { “... we'd be pleased... } \\
\text {... if at least once...” } \\
{[19 \text { quotations }]}\end{array}$} & Expression of XX's fear, because she doesn't feel safe \\
\hline & Insignificant (just a normal office communication) \\
\hline & Complaint/claim \\
\hline & Reprimand/reproach, by XX to YY \\
\hline & XX's clarification request \\
\hline & Information exchange \\
\hline
\end{tabular}




\section{Table $\mathbf{1 1}$ (on next page)}

Examples of possible meta-information stimulus-factors.

The table displays examples, drawn from the filled questionnaires, of a special stimulusfactor inside the messages. The capability of these factors to work as stimuli is not linked to the information they might contain, but to "the fact that" they are present within the message, in a certain form and/or at a certain point (in such sense they represent metainformation to which readers can automatically react). 


\begin{tabular}{|l|l|}
\hline Factors & Examples \\
\hline \hline Form of address & Using or not titles indicates formality level \\
\hline Use of idiomatic expressions & Sign of familiarity, informality \\
\hline Regards / greetings form & $\begin{array}{l}\text { Length and presencelabsence of thanks are taken into } \\
\text { ccount and interpreted as sign of attention, } \\
\text { carelessness, respect, defiance... }\end{array}$ \\
\hline Reply quickness & Courtesy / promptness sign \\
\hline Use of technical terms & Sign of intention to keep a distant role \\
\hline Amount / level of details provided & Sign of major / minor accuracy or interest \\
\hline Quantifying information & Sign of quibbling, coldness \\
\hline Referring to rules / laws & Taken as sign of escalation in formality \\
\hline
\end{tabular}




\section{Table $\mathbf{1 2}$ (on next page)}

Statistical distribution of the answers to the Final question (choice between the "Hard"/H or "Softer"/S version of Message \#4).

The table displays (for the total sample and the two control sub-samples) the frequencies of the answers to the Final question, i.e. the choice between the "Hard" $(H)$ version of Message \#4 and the "Softer" (S) one as the solution of the case. A strong imbalance is shown, as indications of Message \#4/S overwhelm the Message \#4/H ones in all cases. 


\begin{tabular}{|l||l|l|l|l||l|l||}
\hline \multirow{2}{*}{ Variable } & \multicolumn{2}{|l||}{ Total Sample } & \multicolumn{2}{l||}{ Sub-sample AGE } & \multicolumn{2}{l||}{ Sub-sample EMPLOYMENT } \\
\cline { 2 - 8 } & Answers & $\%$ & Answers & $\%$ & Answers & $\%$ \\
\hline "H" choice & 26 & $25.7 \%$ & 16 & $27.6 \%$ & 19 & $30.2 \%$ \\
\hline "S" choice & 75 & $74.3 \%$ & 42 & $72.4 \%$ & 44 & $69.8 \%$ \\
\hline \hline Total & $\mathbf{1 0 1}$ & $100 \%$ & $\mathbf{5 8}$ & $100 \%$ & $\mathbf{6 3}$ & $100 \%$ \\
\hline \hline
\end{tabular}




\section{Table $\mathbf{1 3}$ (on next page)}

Sample distribution with respect to coherence levels.

The table displays, for the total sample and the two sub-samples "Age" and "Employment", the distribution of participants with respect to coherence levels (see text for concept details; see SI, Section 11a and Table S7 for a display of the scale). The L level results overrepresented with regards to what expected. 


\begin{tabular}{||l||l|l||l|l||l|l||}
\hline \multirow{2}{*}{ Coherence level } & Total sample & \multicolumn{2}{|l||}{ Sub-sample "AGE" } & \multicolumn{2}{l||}{ Sub-sample "Employm." } \\
\cline { 2 - 8 } & Values & $\mathbf{\%}$ & Values & $\mathbf{\%}$ & Values & \% \\
\hline \hline L & 12 & 12.2 & 8 & 14.3 & 9 & 14.8 \\
\hline LM & 9 & 9.2 & 6 & 10.7 & 6 & 9.8 \\
\hline MG & 18 & 18.4 & 8 & 14.3 & 9 & 14.8 \\
\hline G & 59 & 60.2 & 34 & 60.7 & 37 & 60.7 \\
\hline \hline Total & $\mathbf{9 8}$ & $\mathbf{1 0 0 . 0}$ & $\mathbf{5 6}$ & $\mathbf{1 0 0 . 0}$ & $\mathbf{6 1}$ & $\mathbf{1 0 0 . 0}$ \\
\hline \hline
\end{tabular}

LEGEND: $L=$ Low LM = Low-medium, $M G=$ Medium-great, $G=$ Great level of coherence between predictions and choice; $\mathrm{H} / \mathrm{S}=$ Versions of Message \#4; +/- = type of predicted effect (resolution or escalation of the conflict) of the messages on XX. 


\section{Table 14(on next page)}

Sample distribution with respect to coherence levels and expressed choice (total sample).

The table displays (for the total sample) the distribution of participants with respect to coherence crossed with the final choice between the "Hard" $(H)$ and the "Softer" $(S)$ version of Message \#4. Data shows that the imbalance in the Low coherence bin is ascribable to " $\mathrm{H}$ " choosers only. A strong correlation between the two variables "coherence" and "choice" is highlighted: Chi-squared test and Fisher's Exact test return high significance $(p<0.001)$. 


\begin{tabular}{||l|l|l||l|l|l||l|l||}
\hline \multicolumn{2}{|l|}{ "H” Choosers } & "S" Choosers & \multicolumn{2}{l||}{ Total } \\
\hline Coherence level & Values & $\%$ & Coherence level & Values & $\%$ & Values & $\%$ \\
\hline L $(\mathrm{H}-/ \mathrm{S}+)$ & 10 & 41.7 & L $(\mathrm{H}+/ \mathrm{S}-)$ & 2 & 2.7 & 12 & 12.2 \\
\hline LM $(\mathrm{H}-/ \mathrm{S}-)$ & 2 & 8.3 & LM $(\mathrm{H}-/ \mathrm{S}-)$ & 7 & 9.5 & 9 & 9.2 \\
\hline MG $(\mathrm{H}+/ \mathrm{S}+)$ & 5 & 20.8 & MG $(\mathrm{H}+/ \mathrm{S}+)$ & 13 & 17.6 & 18 & 18.4 \\
\hline G $(\mathrm{H}+/ \mathrm{S}-)$ & 7 & 29.2 & G $(\mathrm{H}-/ \mathrm{S}+)$ & 52 & 70.3 & 59 & 60.2 \\
\hline \hline Total & $\mathbf{2 4}$ & $\mathbf{1 0 0 . 0}$ & Total & $\mathbf{7 4}$ & $\mathbf{1 0 0 . 0}$ & $\mathbf{9 8}$ & $\mathbf{1 0 0 . 0}$ \\
\hline
\end{tabular}

LEGEND: $L=$ Low LM = Low-medium, $M G=$ Medium-great, $G=$ Great level of coherence between predictions and choice; $\mathrm{H} / \mathrm{S}=$ Versions of Message \#4; +/- = type of predicted effect (resolution or escalation of the conflict) of the messages on XX. 